\title{
Photoreactivity of condensed acetylene on Titan aerosols analogues
}

Benjamin Fleury ${ }^{1}$, Murthy S. Gudipati ${ }^{1,{ }^{*}}$, Isabelle Couturier-Tamburelli ${ }^{2}$, Nathalie Carrasco ${ }^{3,4}$

${ }^{1}$ Science Division, Jet Propulsion Laboratory, California Institute of Technology, 4800 Oak Grove Drive, Pasadena, California 91109, USA

${ }^{2}$ Aix-Marseille Université, CNRS, PIIM, UMR 7345, 13013 Marseille, France

${ }^{3}$ Université Versailles St-Quentin, Sorbonne Universités, UPMC Univ. Paris 06, CNRS/INSU, LATMOS-IPSL, 11 Blvd. d'Alembert, 78280 Guyancourt, France

${ }^{4}$ Institut Universitaire de France, 103 Bvd St-Michel, 75005 Paris, France

*Corresponding author, e-mail: murthy.gudipati@jpl.nasa.gov

Pages: 28

Figures: 10

Tables: 1 


\section{Abstract}

Volatile organic molecules formed by photochemistry in the upper atmosphere of Titan can undergo condensation as pure ices in the stratosphere and the troposphere as well as condense as ice layers onto the organic aerosols that are visible as the haze layers of Titan. As solar photons penetrate through Titan's atmosphere, shorter-wavelength photons are attenuated and longerwavelength photons make it into the lower altitudes, where aerosols become abundant. We conducted an experimental study to evaluate the long wavelength $(\lambda>300 \mathrm{~nm})$ photo-reactivity of these ices accreted on the Titan aerosol-analogs (also known as tholins) made in the laboratory. We have focused on acetylene, the third most abundant hydrocarbon in Titan's atmosphere (after $\mathrm{CH}_{4}$ and $\mathrm{C}_{2} \mathrm{H}_{6}$ ). Further, acetylene is the most abundant unsaturated hydrocarbon in Titan's atmosphere. Our results indicate that the aerosols can act as activation centers to drive the photoreactivity of acetylene with the aerosols at the accretion interface at wavelengths where acetylene-ice alone does not show photoreactivity. We found that along with photochemistry, photodesorption plays an important role. We observed that about $15 \%$ of the initial acetylene is photodesorbed, with a photodesorption rate of $(2.1 \pm 0.2) \times 10^{-6}$ molecules photon $^{-1}$ at $355 \mathrm{~nm}$. This photodesorption is wavelength-dependent, confirming that it is mediated by the UV absorption of the aerosol analogues, similar to photochemistry. We conclude that the UV-Vis properties of aerosols would determine how they evolve further in Titan's atmosphere and on the surface through photochemical alterations involving longerwavelength photons. Stronger extinction coefficients in the longer wavelength UV-Vis regions $(>300 \mathrm{~nm})$ of the aerosol give higher efficiencies of photodesorption of accreted volatiles as well as photochemical incorporation of unsaturated condensates (such as acetylene) into the aerosols.

Keywords: Titan; Titan, atmosphere; Ices; Photochemistry 


\section{Introduction}

Titan, the largest satellite of Saturn, has a dense atmosphere mainly made of molecular nitrogen and methane, the latter of which varies by $1 \%$ to $5 \%$ as a function of the altitude (Cui et al., 2009; Flasar et al., 2005; Hörst, 2017; Koskinen et al., 2011; Niemann et al., 2010). Dissociation and ionization of these two species by the vacuum ultraviolet (VUV, wavelengths below $200 \mathrm{~nm}$ ) solar photons and the energetic particles of Saturn's magnetosphere initiate complex organic chemistry leading to the formation of volatile organic compounds and solid organic aerosols in Titan's upper atmosphere. Aerosol formation is initiated at about $1000 \mathrm{~km}$, in the ionosphere of Titan (Lavvas et al., 2013; Liang et al., 2007; Waite et al., 2007). At lower altitudes, as the temperature decreases with altitude from the lower stratosphere $(\sim 150 \mathrm{~km})$ towards the tropopause (Fulchignoni et al., 2005), many volatiles can condense to form ice particles or ice accreted on the aerosols (Anderson and Samuelson, 2011; Anderson et al., 2010; Anderson et al., 2016; Barth, 2017; Barth and Toon, 2003; de Kok et al., 2008; Frère et al., 1990; Lavvas et al., 2011a; Mayo and Samuelson, 2005; Raulin and Owen, 2002; Sagan and Reid Thompson, 1984; Samuelson and A. Mayo, 1991; Samuelson et al., 1997). These ice particles and aerosols precipitate and deposit onto the surface, where they should have formed an organic layer over a geological time-scale according to a study performed using a 1D photochemical model (Vuitton et al., 2008). As the condensates and aerosols increase in number at these altitudes, solar photons with shorter-wavelengths (higher energy) are attenuated, leaving only the longer-wavelength $(>300 \mathrm{~nm}$ ) photons to penetrate through. Understanding the photochemical transformations in these ices and aerosols caused by the solar photons that are available in these environments is the principal goal of our work.

There are a number of observations realized with Voyager IRIS and more recently Cassini CIRS and VIMS, which report the detection of ice particles in the stratosphere of Titan. Evidence for dicyanoacetylene $\left(\mathrm{C}_{4} \mathrm{~N}_{2}\right)$ ice was found in IRIS data (Khanna et al., 1987; Samuelson et al., 1997) and CIRS data (Anderson et al., 2016). The presence of cyanoacetylene $\left(\mathrm{HC}_{3} \mathrm{~N}\right)$ was also suggested by IRIS data and laboratory spectra (Coustenis et al., 1999; Khanna, 2005b) before being confirmed by CIRS observations (Anderson et al., 2010). Hydrogencyanide $(\mathrm{HCN})$ ice has been tentatively identified using CIRS observations (Anderson and Samuelson, 2011; Samuelson et al., 2007) before being confirmed with VIMS (de Kok et al., 2014). The 
presence of other ices such as $\mathrm{C}_{2} \mathrm{H}_{5} \mathrm{CN}, \mathrm{C}_{2} \mathrm{H}_{6}$, and $\mathrm{C}_{2} \mathrm{H}_{2}$ has also been proposed based on IRIS data and laboratory spectra (Coustenis et al., 1999; Khanna, 2005a; Khanna, 2005b) but they have not been confirmed.

Presence of ices is also reported at the surface of Titan based on Cassini and ground-based observations. Presence of water ice has been inferred from observation done at NASA's Infrared Telescope Facility (IRTF) (Griffith et al., 2003), using Cassini VIMS data (Griffith et al., 2012; Hayne et al., 2014; McCord et al., 2006) and the DISR instrument onboard the Huygens lander (Rannou et al., 2016; Tomasko et al., 2005). Other condensed ices have been suggested based on Cassini VIMS observations, but their identifications remain uncertain. These ices include $\mathrm{CO}_{2}$ (McCord et al., 2008), $\mathrm{C}_{6} \mathrm{H}_{6}, \mathrm{HC}_{3} \mathrm{~N}$ (Clark et al., 2010) and more recently $\mathrm{C}_{2} \mathrm{H}_{2}$ (Singh et al., 2016).

Acetylene is the third most abundant hydrocarbon present in Titan's atmosphere after $\mathrm{CH}_{4}$ and $\mathrm{C}_{2} \mathrm{H}_{6}$ and condensed acetylene should be particularly abundant on Titan. Further, acetylene is the most abundant unsaturated hydrocarbon in Titan's atmosphere, making it a more plausible candidate for photochemical transformations in the condensed-phase. The $\mathrm{C}_{2} \mathrm{H}_{2}$ mixing ratio ranges from 2 to $6 \mathrm{ppm}_{\mathrm{v}}$ as a function of latitude as measured in the stratosphere by Cassini CIRS (Vinatier et al., 2010). Formation of stratospheric $\mathrm{C}_{2} \mathrm{H}_{2}$ ice particles is predicted by modeling studies with a condensation near $70 \mathrm{~km}$ (Anderson and Samuelson, 2011; Barth, 2017; Lavvas et al., 2011a; Raulin and Owen, 2002; Sagan and Reid Thompson, 1984), but not yet detected in Titan's atmosphere. However, evaporated acetylene has been detected from Titan's surface by the GC-MS instrument of the Huygens probe after its landing (Niemann et al., 2010), suggesting that acetylene ice is present on the surface and also recently confirmed by the Cassini VIMS observation of Titan's surface (Singh et al., 2016).

Because solar photons at longer-wavelengths reach lower altitudes, condensed molecules could undergo further processes driven by those long wavelength photons $(\lambda>300 \mathrm{~nm})$, particularly at and below the lower stratosphere $(<150 \mathrm{~km})$ where temperatures also drop below $150 \mathrm{~K}$, all the way to the surface of Titan (Lavvas et al., 2008). Interaction of these photons with the aerosols and ices could lead to two different processes: (a) photodesorption of the volatile condensate molecules through direct or indirect mechanisms (Thrower et al., 2008), (b) longwavelength photons could induce solid-state photochemistry leading to the formation of covalent 
bonds and new condensed volatiles organic compounds (Anderson et al., 2016) as well as solid organics like aerosols as demonstrated recently in laboratory experiments (Couturier-Tamburelli et al., 2014; Couturier-Tamburelli et al., 2015; Gudipati et al., 2013). One or several layers of ices could cover Titan's aerosols in the atmosphere or at the surface (Anderson et al., 2016; Raulin and Owen, 2002). Hence, photo-induced processes such photochemistry or photodesorption could alter the mixing ratios of volatiles and condensates in the atmosphere of Titan.

In this work, we have used laboratory experiments to study the photo-induced processes involving condensed acetylene in Titan's atmosphere, for photons at $\lambda>300 \mathrm{~nm}$ that reach the lower-stratosphere $(<150 \mathrm{~km})$ of Titan. We investigated the interaction between Titan's aerosols accreted with acetylene ice-films using laboratory analogs of Titan aerosols, called "tholins", that are made through cold-plasma discharge of $\mathrm{N}_{2}$ and $\mathrm{CH}_{4}$ gas mixture (Szopa et al., 2006). We use "tholins" to generally describe Titan's organic aerosol analogs made in the laboratory environment using plasma discharge, in agreement with literature usage (Cable et al., 2012).

\section{Experimental methods and protocols}

\subsection{Tholin samples production}

Tholin samples used in this study were produced with the PAMPRE experimental set-up, which simulates aerosol production in Titan's atmosphere (Sciamma-O'Brien et al., 2010; Szopa et al., 2006). A $\mathrm{N}_{2}-\mathrm{CH}_{4}$ gas mixture was subjected to radio-frequency discharge initiating a cold plasma at low pressures $(0.9 \mathrm{mbar})$ and room temperature. Tholins accumulate on sapphire substrates ( $25 \mathrm{~mm}$ of diameter, $2 \mathrm{~mm}$ of thickness) disposed on the grounded electrode and form thin-film deposits. The percentage of methane used to produce the tholin samples is known to influence their compositions (Gautier et al., 2012) and their optical properties (Mahjoub et al., 2012). In order to study the effect of the tholins composition and optical properties on the acetylene photo-reactivity, we have produced tholins with $1 \%$ and $5 \%$ of $\mathrm{CH}_{4}$ diluted in $\mathrm{N}_{2}$, in agreement with the methane concentration profile in the atmosphere of Titan (Hörst, 2017): We call the tholins produced by using the $1 \% \mathrm{CH}_{4}$ as $\mathrm{T}_{1}$-tholin from now onwards. Accordingly, $\mathrm{T}_{5}-$ tholin was obtained using the $5 \%$ methane. For a 2 -hour plasma duration, the thickness of the 
tholin films varied between $630 \mathrm{~nm}$ and $910 \mathrm{~nm}$ based on the initial methane percentage (Mahjoub et al., 2012).

\subsection{Experimental setup}

The photoreactivity of solid acetylene was studied at JPL with an experimental setup that we have named the "TOAST" (Titan Organic Aerosol SpecTroscopy) setup (Couturier-Tamburelli et al., 2014; Gudipati et al., 2013). A schematic diagram of the TOAST setup is presented in Figure 1. It is composed of a vacuum chamber connected to a turbomolecular pump assuring an ultrahigh vacuum of $3 \times 10^{-8}$ mbar as measured by an inverted magnetron gauge at room temperature.

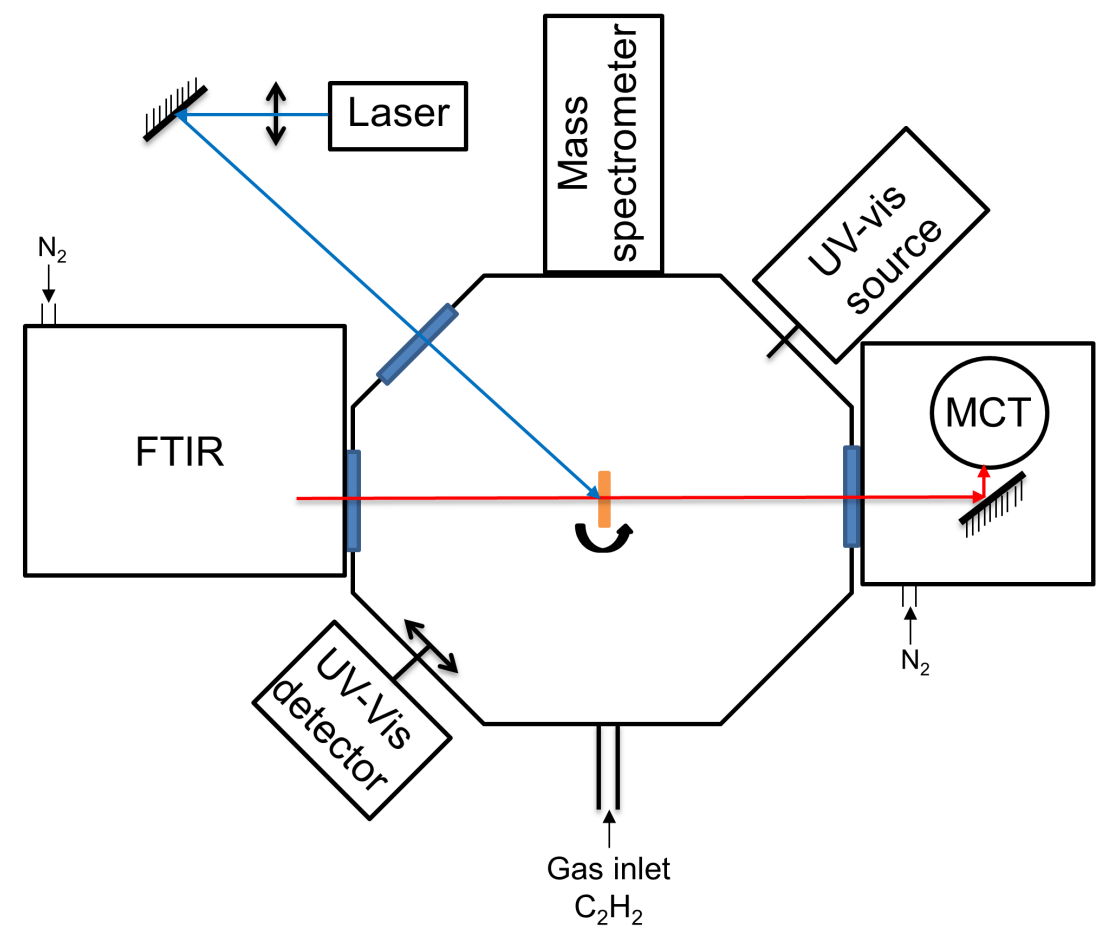

Figure 1: Scheme of the TOAST experimental setup at JPL to simulate and study Titan's atmospheric and surface photochemistry.

Acetylene is highly reactive and easily polymerizes at ambient conditions when stored for a long time. For this reason, we used a gas cylinder of 10\% acetylene diluted in helium (Airgas) at 13 bar total pressure, where acetylene is stabilized over a long period of time (for years), and this gas mixture was deposited on a sapphire window (or a sapphire window coated with a tholin film), cooled down to $50 \mathrm{~K}$ using an ARS closed-cycle helium cryostat. Background pressure during the deposition in the vacuum chamber was kept at $1 \times 10^{-6} \mathrm{mbar}$ and the typical duration of 
deposition was for $20 \mathrm{~min}$. At this temperature, helium does not stick to the sapphire window or the tholin film and it is pumped off, leaving pure acetylene sticking to the sample. Control experiments with pure acetylene ice indicated that acetylene films start significantly sublimating at $70 \mathrm{~K}$ and completely sublime at $80 \mathrm{~K}$ from the sapphire window under the typically used background vacuum of $\sim 5 \times 10^{-9}$ mbar. We chose to keep the temperature at $50 \mathrm{~K}$ for all the experiments, which is lower than the temperature in Titan's atmosphere where acetylene would condense (Fulchignoni et al., 2005), because the pressure at which the experiments were performed (i.e. $\sim 5 \times 10^{-9}$ mbar) which was, by necessity, lower than that found in Titan's stratosphere, would cause thermal sublimation of acetylene. Control experiments confirmed that there was no detectable depletion of infrared absorption bands of acetylene ice over several hours at $50 \mathrm{~K}$. It has been shown that during the warm up of amorphous $\mathrm{C}_{2} \mathrm{H}_{2}$ deposited at $<20 \mathrm{~K}$, amorphous-crystalline phase transition occurred at 45-50 K (Hudson et al., 2014; Knez et al., 2012). Therefore, under our experimental conditions and according to the position and shape of the $v_{3}$ band of $\mathrm{C}_{2} \mathrm{H}_{2}$ presented in Figure 2, we assume that acetylene ice is predominantly in a crystalline form. 


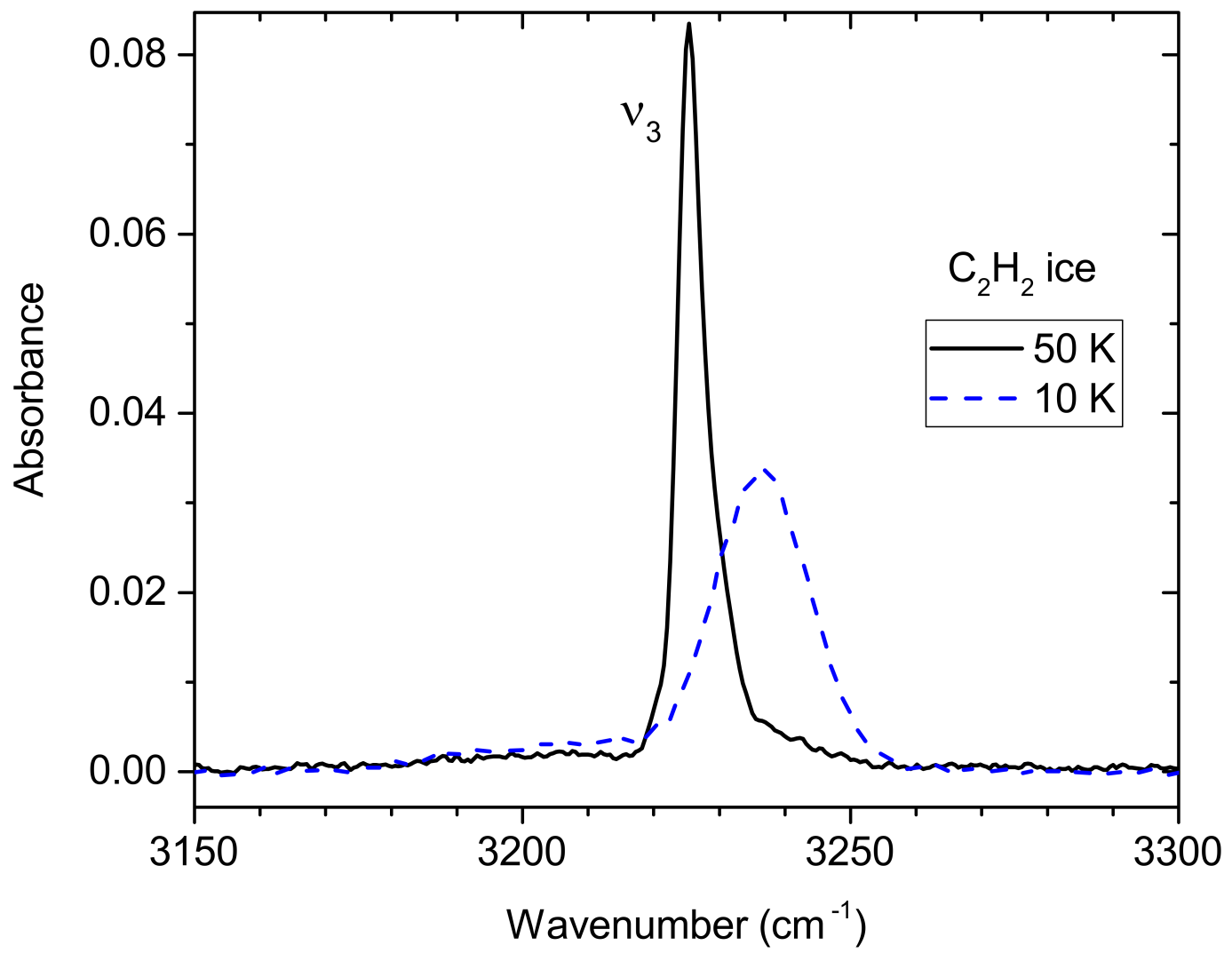

Figure 2: $\mathrm{C}_{2} \mathrm{H}_{2}$ absorption band at $3225 \mathrm{~cm}^{-1}\left(v_{3}\right)$ after $20 \mathrm{~min}$ deposition at $50 \mathrm{~K}$ (crystalline) vs. at $10 \mathrm{~K}$ (amorphous).

Subsequent to the preparation of the acetylene film on sapphire or sapphire with tholin film, this sample was irradiated at different wavelengths to simulate aerosol interaction with solar photons reaching the lower atmosphere of Titan $(<150 \mathrm{~km}): 355 \mathrm{~nm}, 410 \mathrm{~nm}$ and $450 \mathrm{~nm}$. These wavelengths were generated by a Nd:YAG laser (Quantel) with third harmonic generation (355 $\mathrm{nm}$ ) and by tuning an optical parametric oscillator (OPO) (Opotek Opolette-UV) that provides continuous wavelengths between $210 \mathrm{~nm}$ and $700 \mathrm{~nm}$. In order to avoid multi-photon processes and to irradiate the entire sample's surface, the initial laser beam of $3 \mathrm{~mm}$ of diameter was expanded to $20 \mathrm{~mm}$. For $355 \mathrm{~nm}$, the laser beam, which is circular, was expanded using a planoconvex lens ( $75 \mathrm{~mm}$ focal length). For the $410 \mathrm{~nm}$ and $450 \mathrm{~nm}$, the OPO-laser beam that is highly elliptical ( $2 \mathrm{~mm} \times 5 \mathrm{~mm}$ at $10 \mathrm{~cm}$ from the laser exit), was expanded using two planoconvex cylindrical lenses (100 $\mathrm{mm}$ and $200 \mathrm{~mm}$ focal lengths). Photon flux was measured at the entry of the vacuum chamber, after beam expansion. The calculated photon fluxes reaching the sample for each wavelength are given in Table 1. 
Table 1: Photon fluxes reaching the sample at different selected laser wavelength.

\begin{tabular}{|c|c|c|c|}
\hline Laser wavelength $(\mathrm{nm})$ & 355 & 410 & 450 \\
\hline $\begin{array}{c}\text { Photon flux } \\
\left(\text { photons } \cdot \mathrm{cm}^{-2} \cdot \mathrm{s}^{-1}\right)\end{array}$ & $(1.80 \pm 0.05) \times 10^{17}$ & $(2.50 \pm 0.05) \times 10^{16}$ & $(2.70 \pm 0.05) \times 10^{16}$ \\
\hline
\end{tabular}

Infrared (FTIR) and UV-Vis spectroscopy in transmission mode were used to monitor the evolution of acetylene/tholin samples during laser irradiation. Photodesorption of acetylene was monitored using in situ mass spectrometry.

\subsection{Infrared absorption spectroscopy}

Single-beam infrared spectra of the samples were measured with a Thermo Scientific Nicolet 6700 Fourier Transform Infrared Spectrometer (FTIR), which are processed to obtain absorption spectra by choosing appropriate spectra as reference and sample. As shown in Figure 1, the beam enters in the vacuum chamber through a $\mathrm{ZnSe}$ window, goes out through a second $\mathrm{ZnSe}$ window and is focused on to a Mercury Cadmium Telluride (MCT) detector cooled to $77 \mathrm{~K}$ by liquid nitrogen. Infrared spectra were recorded between $1600 \mathrm{~cm}^{-1}$ (sapphire window absorption limit) and $4500 \mathrm{~cm}^{-1}$ at $1 \mathrm{~cm}^{-1}$ resolution by averaging 200 scans.

\subsection{UV-visible absorption spectroscopy}

The UV/visible spectra were measured at a $45^{\circ}$ incidence angle to the sample window (Figure 1), using an Ocean Optics DH-2000 fiber coupled deuterium-halogen source. The output light was focused by a lens and collected by an Ocean Optics USB4000 fiber coupled spectrograph. Spectra were acquired in the 220-1100 nm range by averaging 500 scans recorded with an integration time of $20 \mathrm{~ms}$ per scan.

\subsection{Mass spectrometry analysis of the gas-phase molecules}

In situ measurements of photon-induced desorption of acetylene were carried out using a Stanford Research System RGA200 quadrupole mass spectrometer (QMS) equipped with electron-multiplier to increase sensitivity, which was directly connected to the main vacuum chamber with the sample. QMS ionization was achieved through electron impact at $70 \mathrm{eV}$. It covers 1 to $200 \mathrm{~m} / \mathrm{z}$ mass range with a resolution of 100 at $m / z 100(\mathrm{~m} / \Delta \mathrm{m})$. The QMS measures overall equilibrated content of rest gases in the vacuum chamber. During the thermal and photodesorption experiments, the turbomolecular pump continuously removed gases in the 
vacuum chamber. Thus, the detected ion currents are quantitative and directly comparable between experiments as discussed below.

\subsection{Quantification of $\mathrm{C}_{2} \mathrm{H}_{2}$ Ice Desorption}

The total ion current measured at $m / z 26\left(\mathrm{C}_{2} \mathrm{H}_{2}{ }^{+}\right)$during the irradiation experiments is proportional to the number of acetylene molecules desorbed. In order to calculate the fraction of $\mathrm{C}_{2} \mathrm{H}_{2}$ molecules photodesorbed relative to the initial number of molecules $\mathrm{N}_{0}$ that were deposited, we have first measured the total ion current at $\mathrm{m} / \mathrm{z} 26$ corresponding to the thermal desorption of the entire acetylene deposited. The same amount of acetylene as used in the irradiation experiments has been desorbed using temperature-programmed desorption (TPD) at 1 $\mathrm{K} \cdot \mathrm{min}^{-1}$. The TPD curve measured for $\mathrm{C}_{2} \mathrm{H}_{2}$ is presented in Figure 3. The amount of acetylene deposited for each experiment is controlled using the absorbance of the $3225 \mathrm{~cm}^{-1}$ band, which is proportional to the thickness of the ice deposited. The thickness of the deposited acetylene ice has been calculated using the Eq. (1):

$h=\frac{\int \tau_{v} d v}{A \times \rho_{N}}$

where $\int \tau_{v} d v$ is the integral of the band in optical depth, which can be derived from the absorbance value. $A$ is the band-strength $\left(\mathrm{cm}\right.$. molecule $^{-1}$ ) and $\rho_{\mathrm{N}}$ the number density $\left(\right.$ molecules. $\mathrm{cm}^{-3}$ ). For our calculation, we used a band strength value of $3.58 \times 10^{-17} \mathrm{~cm} \cdot$ molecule $^{-}$

${ }^{1}$ for the $3225 \mathrm{~cm}^{-1}$ band calculated by Hudson et al. (2014) for the crystalline acetylene at $70 \mathrm{~K}$. We used a number density of $1.76 \times 10^{22}$ molecules $\cdot \mathrm{cm}^{-3}$ derived from the mass density, assuming a value of $0.76 \mathrm{~g} \cdot \mathrm{cm}^{-3}$ (Hudson et al., 2014; McMullan et al., 1992), Avogadro's number and the molar mass of acetylene $\left(26.04 \mathrm{~g} \cdot \mathrm{mol}^{-1}\right)$. We calculated an average thickness of the acetylene ice film to be between $15 \mathrm{~nm}$ and $20 \mathrm{~nm}$, based on minor variations in the deposition conditions that reflect in acetylene absorbance at $3225 \mathrm{~cm}^{-1}$. 


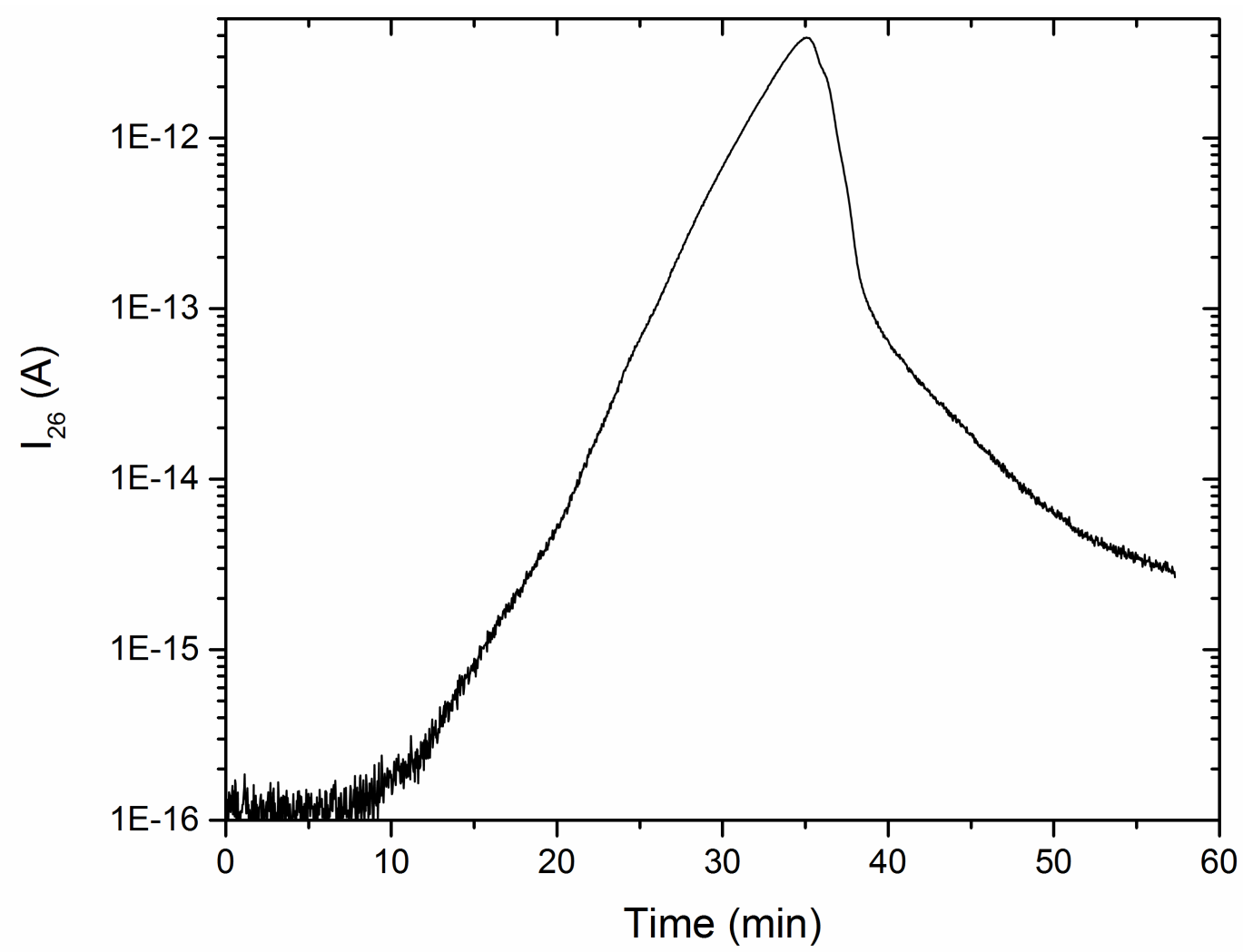

Figure 3: Evolution of $m / z=26\left(\mathrm{C}_{2} \mathrm{H}_{2}{ }^{+}\right)$intensity as function of time during the temperature-programmed desorption (TPD) of a $\mathrm{C}_{2} \mathrm{H}_{2}$ ice film prepared at $50 \mathrm{~K}$.

The integrated signal at $m / z 26$ measured during the TPD is proportional to the amount of desorbed $\mathrm{C}_{2} \mathrm{H}_{2}$ molecules $\mathrm{N}_{0}$, which is used to calculate the percentage of acetylene photodesorbed during the laser irradiation. Compared to TPD, which is a continuous process, laser irradiation at $20 \mathrm{~Hz}$ repetition rate is a pulsed excitation (5 ns laser pulse width), which is expected to generate photodesorption within a shorter period of time in microseconds range, until the next laser pulse comes 50 milliseconds later. In order to ensure that such pulsed ejection of acetylene into the vacuum chamber is measured accurately, we integrated the ion-current at each mass for 400 milliseconds, averaging 8 laser pulses. Four mass peaks were monitored through this process, namely: $\mathrm{H}_{2} \mathrm{O}^{+}(m / z 18), \mathrm{C}_{2} \mathrm{H}^{+}(m / z 25), \mathrm{C}_{2} \mathrm{H}_{2}{ }^{+}(m / z 26)$ and $\mathrm{O}_{2}{ }^{+}(m / z ~ 32)$. Thus, our laser photodesorption data can be quantitatively compared with TPD data for acetylene. 


\section{Results}

\subsection{Acetylene photo-reactivity experiments}

\subsubsection{Pure $\mathrm{C}_{2} \mathrm{H}_{2}$-ice and $\mathrm{C}_{2} \mathrm{H}_{2}$ on $\mathrm{T}_{1}$-tholin}

We began our study with the photo-reactivity of acetylene deposited on a sapphire window or a $\mathrm{T}_{1}$-tholin sample and irradiation with a laser at $355 \mathrm{~nm}$. We have chosen to first focus on $\mathrm{T}_{1}$ tholin, which has higher nitrogen incorporation than the $\mathrm{T}_{5}$-tholin. The evolution of the acetylene amount on the sample was monitored using its infrared band at $3225 \mathrm{~cm}^{-1}$, attributed to the $v_{3}$ vibrational stretching mode of the C-H bond (Hudson et al., 2014).

Figure 4 presents the evolution of the differential absorbance after each irradiation for pure $\mathrm{C}_{2} \mathrm{H}_{2}$ in the left panel and for $\mathrm{C}_{2} \mathrm{H}_{2}$ deposited on a $\mathrm{T}_{1}$-tholin sample in the right panel. The differential absorbance is the absorbance after a time " $t$ " of irradiation $A_{t}$ less the initial absorbance $A_{t=0}$. Figure 4 shows a decrease of the acetylene absorbance after irradiation at $355 \mathrm{~nm}$, indicating depletion of acetylene in the sample. This photo-depletion is enhanced by an order of magnitude when acetylene was deposited on a $\mathrm{T}_{1}$-tholin compared to the pure $\mathrm{C}_{2} \mathrm{H}_{2}$-ice on the sapphire substrate experiment. 


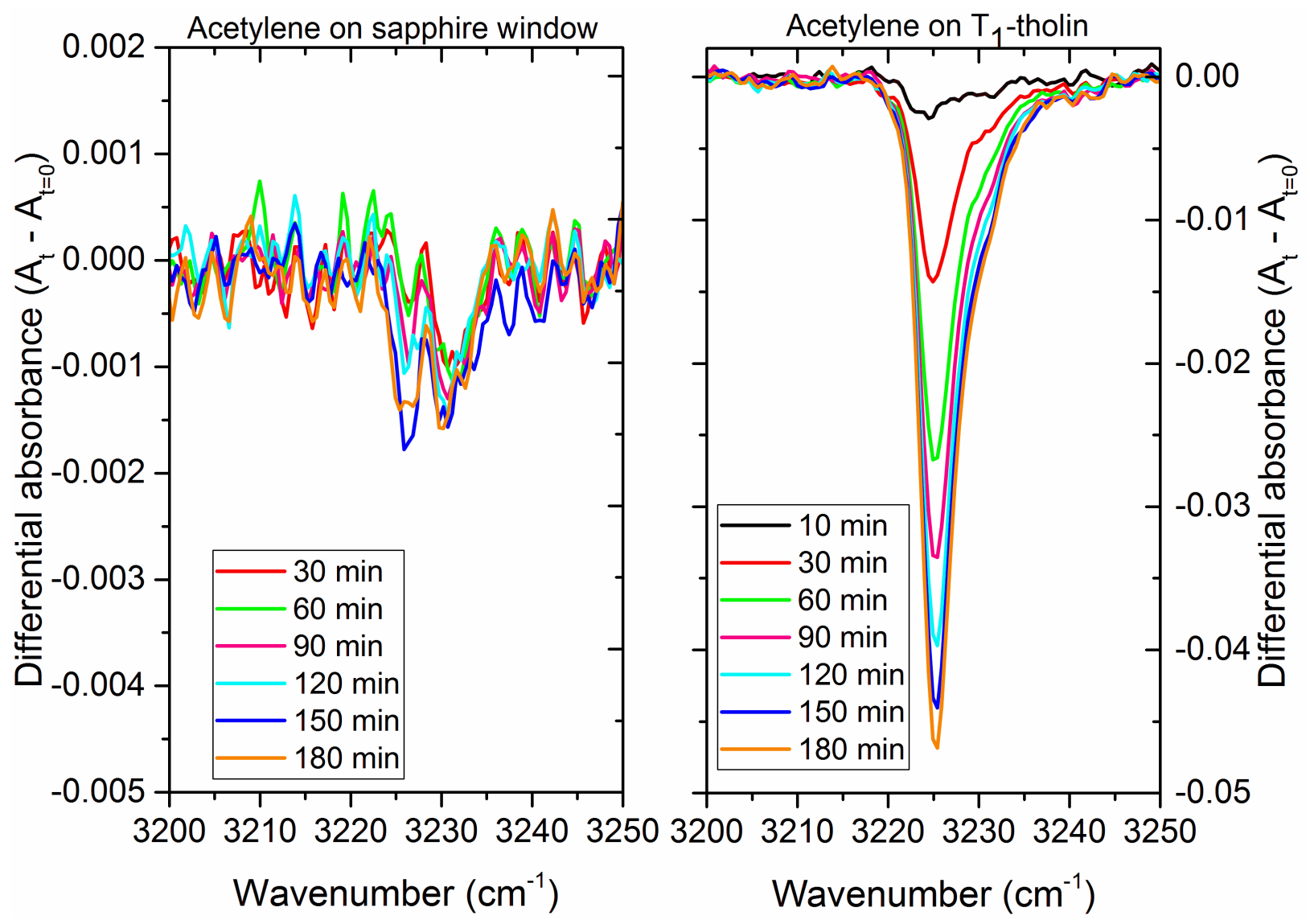

Figure 4: Evolution of the differential absorbance $\left(A_{t}-A_{t=0}\right)$ of $\mathrm{C}_{2} \mathrm{H}_{2}$ as a function of the irradiation duration at $355 \mathrm{~nm}$. The left panel presents results for acetylene coated on blank sapphire window. The right panel presents results for acetylene coated on a $T_{1}$-tholin sample.

Two possible photo-induced processes can be considered to explain the observed acetylene's depletion, namely, photodesorption and photochemistry. The photodesorption of $\mathrm{C}_{2} \mathrm{H}_{2}$ was monitored by in situ mass spectrometry using the time-tracking of $\mathrm{C}_{2} \mathrm{H}_{2}{ }^{+}$at $\mathrm{m} / \mathrm{z} 26$ in the residual gas phase during the irradiation. Figure 5 presents the evolution of the ion current measured by the mass spectrometer for $m / z 26$ as function of time during the irradiation at $355 \mathrm{~nm}$ of $\mathrm{C}_{2} \mathrm{H}_{2}$ on a $\mathrm{T}_{1}$-tholin sample. When the laser was turned on, we observed an increase of the signal by two orders of magnitude. The intensity decreased slowly during the irradiation and then returned to its initial value when the laser was turned off. This increase of the signal measured by the QMS during the irradiation highlights the photodesorption of acetylene during the irradiation at 355 $\mathrm{nm}$. 


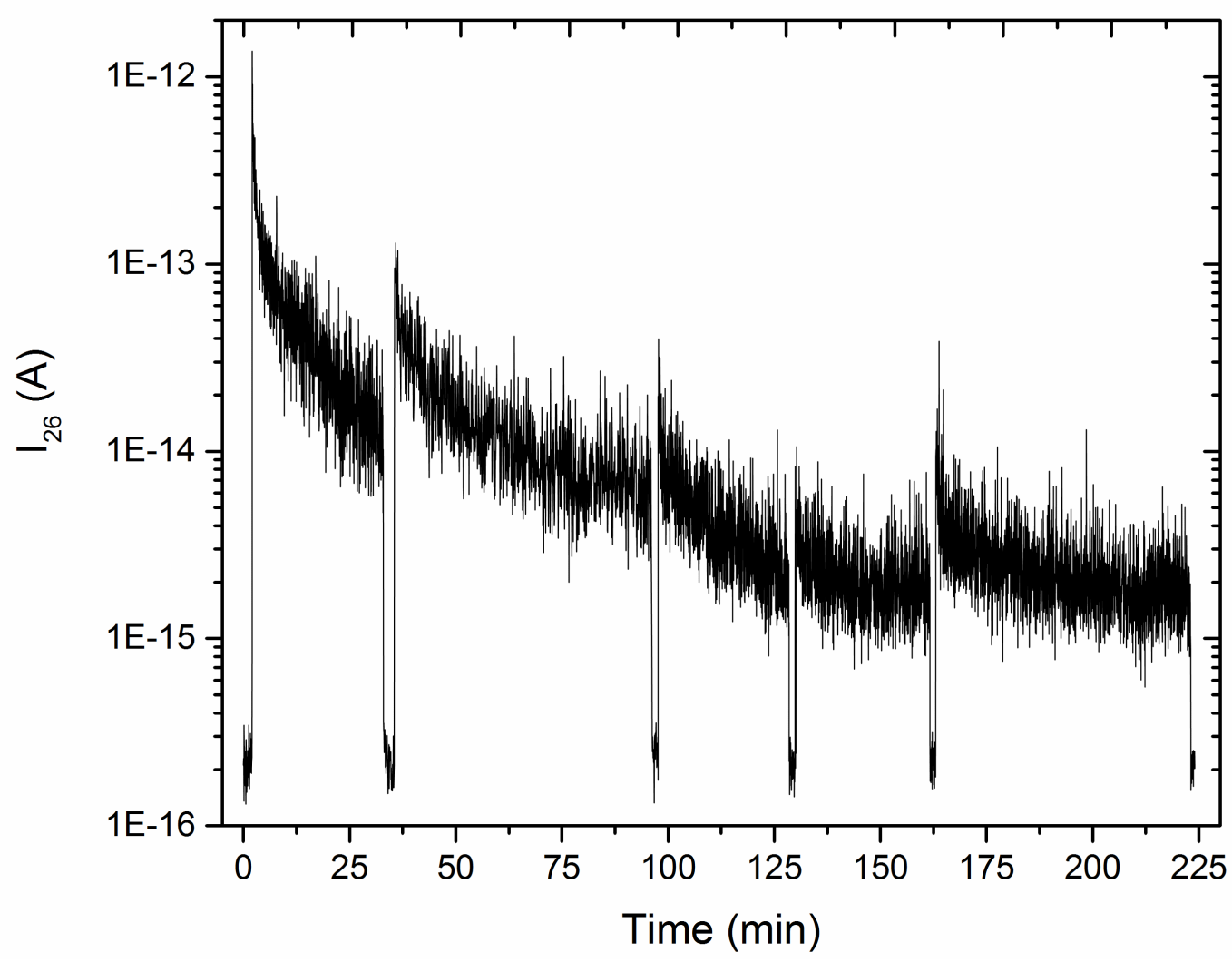

Figure 5: Evolution of $m / z=26\left(\mathrm{C}_{2} \mathrm{H}_{2}{ }^{+}\right)$intensity as function of the time for the different irradiations step at $355 \mathrm{~nm}$ of $\mathrm{C}_{2} \mathrm{H}_{2}$ deposited on a $\mathrm{T}_{1}$-tholin sample. Origin of time is set as the moment when the laser was turned on for the first time. Interruptions are the timewindows when IR were measured.

We have measured the integrated signal for each irradiation and calculated the fraction of the initial amount of acetylene photodesorbed for each irradiation (presented in Section 2.6). Similarly, we have calculated for each irradiation the amount of acetylene consumed, using the integrated band area at $3225 \mathrm{~cm}^{-1}$ in the IR absorption spectrum. With this procedure, we can compare the evolution of the acetylene amount photodesorbed, measured with the QMS and remaining as ice film, measured using IR spectroscopy. Figure 6 presents the evolution of the amount of acetylene as a function of the irradiation time as measured by IR spectroscopy as well as the evolution of gas-phase acetylene quantity taking into account the acetylene desorbed as measured by QMS. Please note that due to negligible photodesorption of pure acetylene ice from the sapphire window, we have only quantified photodesorption for the experiment where acetylene was deposited on the $\mathrm{T}_{1}$-tholin sample. 


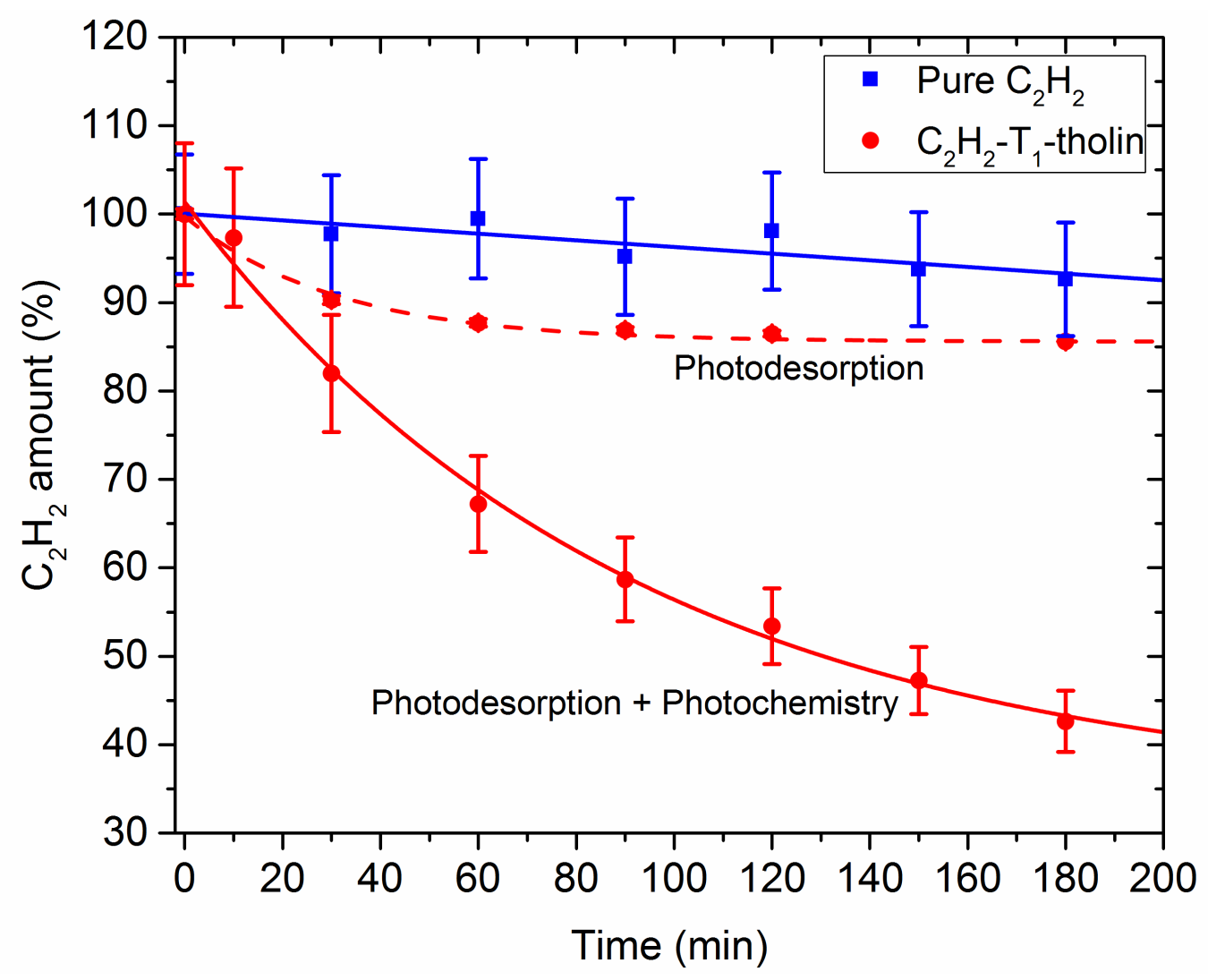

Figure 6: Evolution of the $\mathrm{C}_{2} \mathrm{H}_{2}$ amount as a function of the irradiation time measured by infrared spectroscopy (solid line) for pure $\mathrm{C}_{2} \mathrm{H}_{2}$-ice and for $\mathrm{C}_{2} \mathrm{H}_{2}$ deposited on a $\mathrm{T}_{1}$-tholin sample. Evolution of the $\mathrm{C}_{2} \mathrm{H}_{2}$ amount as a function of the irradiation time for the photodesorption process measured by mass spectrometry for the $\mathrm{C}_{2} \mathrm{H}_{2}$ - $\mathbf{T}_{1}$-tholin experiment (dash line). The uncertainties are given at $2 \sigma$ (standard deviation) and were calculated from the standard fluctuations of the infrared spectroscopy and mass spectrometry measurements, respectively.

We found that in the pure $\mathrm{C}_{2} \mathrm{H}_{2}$-ice experiment, the acetylene consumption follows linear kinetics with a total amount of acetylene consumed about 5\% after 3 hours of irradiation, while in the $\mathrm{C}_{2} \mathrm{H}_{2}$ on $\mathrm{T}_{1}$-tholin experiment the acetylene consumption follows exponential kinetics with a total amount of acetylene consumed about $60 \%$ after 3 hours of irradiation. Figure 6 shows a good correlation for the first 10 minutes of irradiation and then a divergence between these two quantities. Indeed, Figure 6 shows that after 10 minutes of irradiation the total amount of acetylene consumed is still increasing (determined by infrared absorption band integrated intensity at $3225 \mathrm{~cm}^{-1}$ ), while the amount of acetylene photo-desorbed reaches a plateau (measured by QMS). It indicates that the freshly accreted acetylene has higher propensity to photodesorb, whereas aged ice or aerosols desorb far less acetylene. Under our experimental conditions, we found that the acetylene amount consumed by photodesorption was about $15 \%$ 
after 3 hours of irradiation while the total amount of $\mathrm{C}_{2} \mathrm{H}_{2}$ consumed was about $60 \%$. This result highlights that about $25 \%$ of the acetylene was lost to photodesorption in these experiments while the other $75 \%$ depletion was linked to another process, namely photochemistry.

Since solid acetylene has only a weak $\mathrm{S}_{0} \rightarrow \mathrm{T}_{1}$ (spin-forbidden) transition at an energy about $3.58 \mathrm{eV}(346 \mathrm{~nm})$ (Couturier-Tamburelli et al., 2015; Malsch et al., 2001) and the photon energy used during these experiments was about $3.49 \mathrm{eV}(355 \mathrm{~nm})$, Desorption Induced by Electronic Transition (DIET), which has been studied for other ices such as CO (Fayolle et al., 2011), should not be an important process. Further, DIET cannot explain the lack of photodesorption observed in the pure acetylene experiment. We propose an indirect mechanism that could explain the photodesorption of acetylene that occurs selectively when $\mathrm{C}_{2} \mathrm{H}_{2}$ is accreted on $\mathrm{T}_{1}$-tholin but not from the pure $\mathrm{C}_{2} \mathrm{H}_{2}$-ice films. We propose that the photons were primarily absorbed by the $\mathrm{T}_{1}$-tholin and the excitation energy was transferred to acetylene molecules, resulting in electronically and vibrationally excited $\mathrm{C}_{2} \mathrm{H}_{2}$ molecules and thermal desorption of $\mathrm{C}_{2} \mathrm{H}_{2}$ at the surface. Indeed, several studies have highlighted that such a mediated process could occur when one molecule absorbs the photon energy and another molecule desorbs (Bertin et al., 2012; Fillion et al., 2014; Thrower et al., 2008). In our experiments, $\mathrm{T}_{1}$-tholin acted as a very efficient photon absorption and energy transfer system compared to sapphire, which has essentially no photoabsorption at UV-Vis wavelengths. However, a limited acetylene photodesorption mediated by the weak $S_{0} \rightarrow T_{1}$ (spin-forbidden) transition or by the sapphire substrate could occur as also been observed for benzene and water under similar conditions (Thrower et al., 2008). This mechanism could explain the $5 \%$ of acetylene depletion in the pure acetylene experiment. Figure 7 presents the absorbance of the $\mathrm{T}_{1}$-tholin and $\mathrm{T}_{5}$-tholin samples in the 250 $800 \mathrm{~nm}$ range. Tholins do not absorb at $\lambda>500 \mathrm{~nm}$, but the absorption becomes monotonically stronger at wavelengths shorter than $450 \mathrm{~nm}$. In this scenario, the tholin film first absorbs the photons energy and then transfers this energy to the neighboring $\mathrm{C}_{2} \mathrm{H}_{2}$ molecules. Thus, tholins acting as antenna molecules increased the photodesorption efficiency by a factor of 3 at the wavelength considered compared to pure $\mathrm{C}_{2} \mathrm{H}_{2}$ deposited on a sapphire window. Tholin samples are also highly porous (Carrasco et al., 2009) and hence are poor thermal conductors. This property could enable tholins to be electronically excited through the absorption of a photon and convert that energy efficiently into thermal energy, which could be transmitted to acetylene molecules adsorbed in the porous voids, resulting in their photodesorption. 


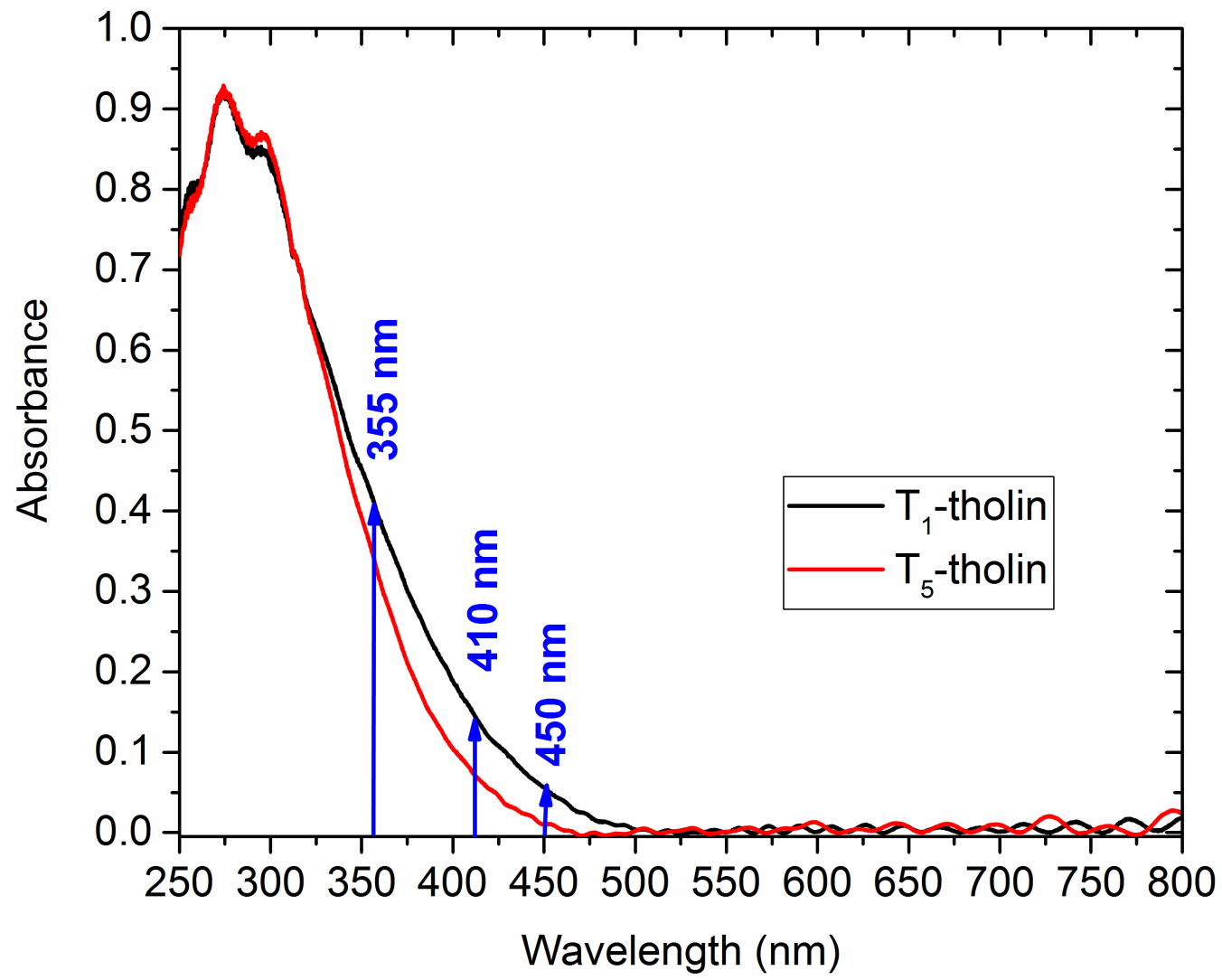

Figure 7: Absorbance of a $T_{1}$-tholin sample and a $T_{5}$-tholin sample (red) in the $250-800 \mathrm{~nm}$ range. The blue arrows point to the different wavelengths of irradiation used during the experiments.

Thus, photodesorption of acetylene mediated by tholins appears to be an important process. However, this process explains only $25 \%$ of the acetylene consumption observed by IR spectroscopy. Most likely, the other process, which is responsible for the other $75 \%$ of the $\mathrm{C}_{2} \mathrm{H}_{2}$ consumption, should be photochemistry. This possibility is further discussed later in the article.

\subsubsection{Wavelength dependence of the $\mathrm{C}_{2} \mathrm{H}_{2}$ photodesorption on $\mathrm{T}_{1}$-tholin}

It has been shown in other studies that the photodesorption processes are wavelength dependent (Bertin et al., 2013; Fillion et al., 2014; Thrower et al., 2008). In our experiments also photodesorption of acetylene was wavelength dependent but driven by the photoabsorption of $\mathrm{T}_{1}$-tholin. This hypothesis is further corroborated by the dependence of the acetylene consumption from the $\mathrm{C}_{2} \mathrm{H}_{2}$-over- $\mathrm{T}_{1}$-tholin sample as a function of the wavelength of irradiation at $410 \mathrm{~nm}$ and $450 \mathrm{~nm}$. To compensate for the lower flux of photons at $410 \mathrm{~nm}$ and $450 \mathrm{~nm}$ compared to $355 \mathrm{~nm}$ as shown in Table 1, we irradiated the sample for longer times at these two wavelengths, 29 hours and 25 hours, respectively, resulting in similar total photon fluence at all 
three wavelengths ( $355 \mathrm{~nm}, 410 \mathrm{~nm}$, and $450 \mathrm{~nm}$ ). It is important to note here that we have not detected desorption of acetylene using mass spectrometry at $410 \mathrm{~nm}$ and $450 \mathrm{~nm}$. However, we have measured a decrease of the IR absorbance of $\mathrm{C}_{2} \mathrm{H}_{2}$ as discussed previously for the $355 \mathrm{~nm}$ irradiation. Figure 8 presents the evolution of acetylene concentration, monitored with IR spectroscopy, as a function of the photon-fluence for $\mathrm{C}_{2} \mathrm{H}_{2}$-over- $\mathrm{T}_{1}$-tholin sample for the experiments at $355 \mathrm{~nm}, 410 \mathrm{~nm}$ and $450 \mathrm{~nm}$. Also included here are the total acetylene depletion data (measured by IR spectroscopy) and the depletion data that were due to the photodesorption (measured by mass spectrometry) at $355 \mathrm{~nm}$ for comparison.

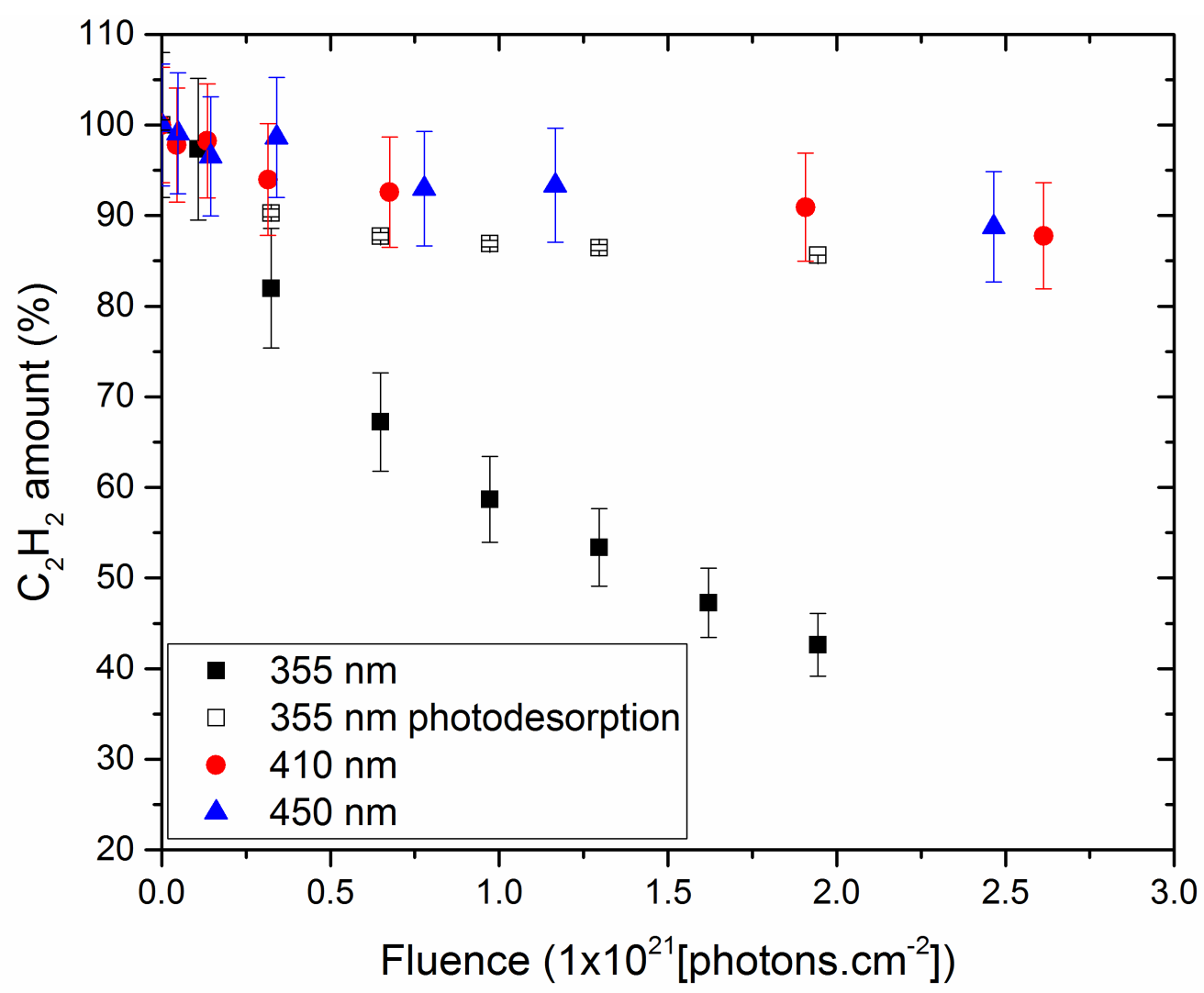

Figure 8: Evolution of the $\mathrm{C}_{2} \mathrm{H}_{2}$ amount measured by infrared spectroscopy for $\mathrm{C}_{2} \mathrm{H}_{2}$ deposited on a $T_{1}$-tholin sample as a function of the photon-fluence at $355 \mathrm{~nm}$ (black square), $410 \mathrm{~nm}$ (red circle) and $450 \mathrm{~nm}$ (blue triangle). Photodesorption of $\mathrm{C}_{2} \mathrm{H}_{2}$ at $355 \mathrm{~nm}$ was quantified by mass spectrometry whereas the rest of the curves are quantified through infrared absorption, which has much larger error bars in integrated absorbance. The uncertainties are given at $\mathbf{2} \sigma$ (standard deviation) and were calculated from the standard fluctuations of the infrared spectroscopy and mass spectrometry measurements respectively.

The total amount of acetylene consumed is similar for $410 \mathrm{~nm}$ and $450 \mathrm{~nm}$ with a maximum about $10 \%$ after an irradiation with a fluence $\sim 2.5 \times 10^{21}$ photons $\cdot \mathrm{cm}^{-2}$, which corresponds to a 
total time of irradiation of $\sim 1700 \mathrm{~min}$ and $\sim 1500$ min respectively according to the photons flux given in Table 1 for these two wavelengths. For both wavelengths, the kinetics is slower and the total amount of acetylene consumed was much lower than the $355 \mathrm{~nm}$ irradiations. The UVvisible absorbance spectrum of the $\mathrm{T}_{1}$-tholin presented in Figure 7 shows that tholins still absorb at $410 \mathrm{~nm}$ and $450 \mathrm{~nm}$. Therefore, the amount of acetylene consumed measured by IR spectroscopy at these two wavelengths, can be explained by an indirect photodesorption process mediated by the tholins acting as antenna, as discussed earlier. The lack of detection of acetylene by mass spectrometry in the $410 \mathrm{~nm}$ and $450 \mathrm{~nm}$ experiments may be explained by the detection limit of the mass spectrometer $\left(<2 \times 10^{-16} \mathrm{~A}\right)$, reached due to the much lower photon flux of the laser used at these two wavelengths compared to $355 \mathrm{~nm}$. In addition, the absorption of $\mathrm{T}_{1^{-}}$ tholins is about 3 and 8 times lower at 410 and $450 \mathrm{~nm}$, compared to $355 \mathrm{~nm}$, resulting in 30 to 80 times lower efficiency of energy transfer at these two visible wavelengths. Further, energy transfer from $\mathrm{T}_{1}$-tholin to acetylene becomes even less efficient due to lack of electronic excitations in acetylene at $410 \mathrm{~nm}$ and $450 \mathrm{~nm}$, necessitating energy to be transferred through thermal vibrational modes. Hence, the amount of acetylene photodesorbed over the integrated time of 400 milliseconds at these two wavelengths $(410$ and $450 \mathrm{~nm})$ is below the limit of detection of our mass spectrometer but clearly detectable in IR spectra of the $\mathrm{C}_{2} \mathrm{H}_{2}$-over- $\mathrm{T}_{1}$ tholin sample over the period of time.

\subsubsection{Influence of the tholins composition on the acetylene reactivity}

The results presented in the previous sections highlight that the photoabsorption of tholins at $\lambda<450 \mathrm{~nm}$ could mediate photoinduced processes in an accreted $\mathrm{C}_{2} \mathrm{H}_{2}$-ice layer, leading to indirect photodesorption and possibly photochemistry of acetylene. The experimental parameters, such as the energy source, the pressure or the initial gaseous mixture composition, used to simulate Titan's atmospheric chemistry are known to impact the properties of the tholins produced and their ability to reproduce Titan's aerosols properties (Cable et al., 2011), particularly for their optical properties (Brassé et al., 2015). A previous study by Mahjoub et al. (2012) has shown that the optical indices of the tholins produced in our experimental setup were, in particular, sensitive to the percentage of methane used. Tholins produced in a gaseous mixture with a lower amount of $\mathrm{CH}_{4}$ incorporate more nitrogen resulting in a slightly higher absorbance of the tholins in the UV-visible range (Mahjoub et al., 2012). For this reason, we have also studied the impact of the initial composition of the tholins and their optical properties by 
repeating the experiments that were conducted with $\mathrm{T}_{1}$-tholin, with a tholin sample produced with a higher percentage of $\mathrm{CH}_{4}, 5 \%$ or $\mathrm{T}_{5}$-tholin sample.

First, the comparison of the UV-Vis absorbance spectra of the $T_{1}$ - and the $T_{5}$-tholin samples shows a similar shape with a strong absorption in the UV starting at $500 \mathrm{~nm}$ and increasing for shorter wavelengths. However the $\mathrm{T}_{1}$-tholin sample presents a slightly higher absorbance between $350 \mathrm{~nm}$ and $500 \mathrm{~nm}$ in agreement with the optical indices measured in a previous work (Mahjoub et al., 2012). Second, we compare the reactivity of $\mathrm{C}_{2} \mathrm{H}_{2}$ deposited on $\mathrm{T}_{1}$ - or $\mathrm{T}_{5}$-tholin samples. We found significant differences in the photodepletion of acetylene when accreted over the $\mathrm{T}_{1}$-tholin sample compared to the $\mathrm{T}_{5}$-tholin sample as shown in Figure 9 . We observed that the photo-reactivity of acetylene does not reflect the absorption spectra of $T_{1}$ - and $T_{5}$-tholins (Figure 7), demonstrating that UV-absorption alone was not the determining factor for these differences of reactivity.
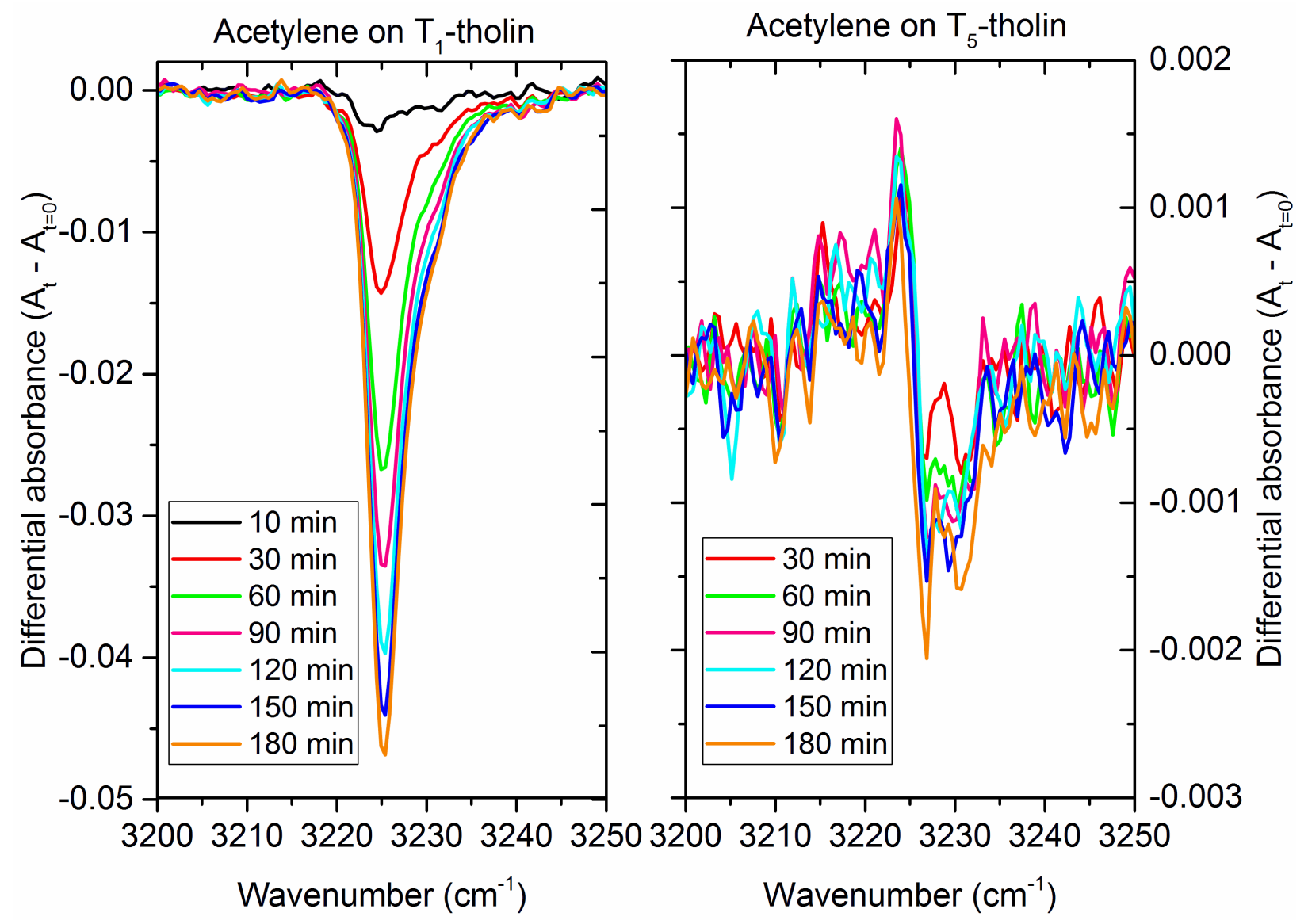

Figure 9: Evolution of the differential absorbance $\left(\mathrm{A}_{t}-\mathrm{A}_{t=0}\right)$ of $\mathrm{C}_{2} \mathrm{H}_{2}$ as a function of the irradiation duration at $355 \mathrm{~nm}$. The left panel presents results for acetylene coated on a 
PAMPRE tholin sample produced in a gaseous mixture made of $1 \%$ of $\mathrm{CH}_{4}$ in $\mathrm{N}_{2}\left(\mathrm{C}_{2} \mathrm{H}_{2}-\right.$ over- $T_{1}$-tholin). The right panel presents results for acetylene coated on a PAMPRE tholin sample produced in a gaseous mixture made of $5 \%$ of $\mathrm{CH}_{4}$ in $\mathrm{N}_{2}\left(\mathrm{C}_{2} \mathrm{H}_{2}\right.$-over- $\mathrm{T}_{5}$-tholin).

Though we detected photodepletion of $\mathrm{C}_{2} \mathrm{H}_{2}$ in the experiments carried out with the $\mathrm{T}_{5}$-tholin samples, photochemistry involving acetylene is found to be inefficient compared to the $\mathrm{T}_{1}$-tholin sample. The total depletion of $\mathrm{C}_{2} \mathrm{H}_{2}$ after three hours of irradiation amounts to $6 \%$ for the $\mathrm{T}_{5}$ tholin experiment, an order of magnitude lower than $60 \%$ observed for the $\mathrm{T}_{1}$-tholin experiment, though the fluence of the $355 \mathrm{~nm}$ laser was same in both experiments. The depletion of acetylene ice on $\mathrm{T}_{5}$-tholin is similar to the result obtained in the pure acetylene experiment or with $\mathrm{T}_{1^{-}}$ tholin at $410 \mathrm{~nm}$ and $450 \mathrm{~nm}$. We attribute the lack of photochemistry of the $\mathrm{C}_{2} \mathrm{H}_{2}$-over- $\mathrm{T}_{5}$-tholin sample as due to the chemical composition difference between $\mathrm{T}_{1^{-}}$and $\mathrm{T}_{5}$-tholins. Indeed, $\mathrm{T}_{1^{-}}$tholins incorporate more nitrogen than $\mathrm{T}_{5}$-tholins (Gautier et al., 2014; Sciamma-O'Brien et al., 2010), leading to tholins enriched in amines (Gautier et al., 2012) for $T_{1}$-tholin. Increased absorption between 400 and $500 \mathrm{~nm}$ of $\mathrm{T}_{1}$-tholin (Figure 7) also indicates higher "unsaturated covalent bonding" in $\mathrm{T}_{1}$-tholin compared to $\mathrm{T}_{5}$-tholin. This difference of composition could influence the indirect photodesorption as well as photochemistry of the acetylene ice. Indeed, the efficiency of this mechanism depends on the nature of interaction between the ice and the UV absorber as well as the possibility for the absorber (tholin) to transfer energy to acetylene (and eventually lead to chemical bonding between acetylene and tholin). The higher the amine and unsaturated bonding in the tholin sample, the higher would be the probability of formation of covalent bonding between tholin and acetylene mediated by soft-excitations with longer wavelength photons. As a result, the efficiency of photodesorption and photochemistry of $\mathrm{C}_{2} \mathrm{H}_{2}$ mediated by $\mathrm{T}_{1}$-tholin films is very significant and these processes are very likely to occur in Titan's atmosphere and on the surface. We conclude that aerosols produced with lesser $\mathrm{CH}_{4}$ mixing with $\mathrm{N}_{2}$ are a potentially important carriers of photochemical sink of acetylene in Titan's atmosphere.

\section{Discussions}

\subsection{Photodesorption rates and implications for Titan}

We can calculate the photodesorption rate of acetylene for our experimental conditions using the following Eq. (2): 
$Y_{P D}=\frac{\mathrm{N}}{\Phi}$,

where $\mathrm{Y}_{\mathrm{PD}}$ is the photodesorption rate (molecules photon $^{-1}$ ), $\mathrm{N}$ is the molecular column density (molecules $\cdot \mathrm{cm}^{-2}$ ) and $\Phi$ is the fluence (photons $\cdot \mathrm{cm}^{-2}$ ). $\mathrm{N}$ can be calculated as the product of the initial column density $\mathrm{N}_{0}$ and the amount of acetylene photodesorbed as measured by mass spectrometry. $\mathrm{N}_{0}$ is calculated from the initial absorbance of $\mathrm{C}_{2} \mathrm{H}_{2}$ before the irradiation using the following Eq. (3):

$N_{0}=\frac{\int \tau_{v} d v}{A}$,

where $\mathrm{N}_{0}$ is the initial column density (molecules $\cdot \mathrm{cm}^{-2}$ ), $\int \tau_{v} d v$ is the integral of the band in optical depth, which can be derived from the absorbance value. A is the band-strength $\left(\mathrm{cm} \cdot\right.$ molecule $\left.^{-1}\right)$. For our calculation, we used a band strength value of $3.58 \times 10^{-17} \mathrm{~cm} \cdot$ molecule $^{-1}$ for the $3225 \mathrm{~cm}^{-1}$ band calculated by Hudson et al. (2014) for the crystalline acetylene at $70 \mathrm{~K}$. In our experiments, the $\mathrm{N}_{0}$ values are found to be $2.89 \times 10^{16}$ molecules $\cdot \mathrm{cm}^{-2}$. We obtained on an average a desorption rate of $(2.1 \pm 0.2) \times 10^{-6}$ molecules photon $^{-1}$ averaged on the total irradiation time at $355 \mathrm{~nm}$. This is much lower than the values determined for the photodesorption of $\mathrm{CO}$, $\mathrm{N}_{2}$ or $\mathrm{CO}_{2}$ ices by high-energy photons $(\lambda<170 \mathrm{~nm})$, which varies from $1 \times 10^{-3}$ to $4 \times 10^{-2}$

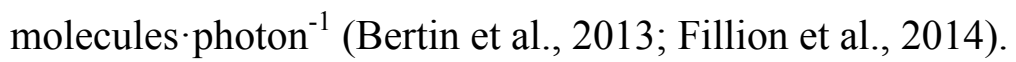

The residence time of the aerosols in the atmosphere of Titan has been calculated using both a 1-D microphysical model (Lavvas et al., 2011b; Lavvas et al., 2010) and a general circulation model (GCM) including the microphysics of aerosols (Larson et al., 2014), based on which it was estimated that the total lifetime of the aerosols in the atmosphere to be 395 Titanian years, which corresponds to $\sim 8.9 \times 10^{9} \mathrm{~s}$. For the wavelength of $350 \mathrm{~nm}$, the photon flux in the lower atmosphere of Titan varies from $\sim 3 \times 10^{11}$ photons $\cdot \mathrm{cm}^{-2} \cdot \mathrm{s}^{-1}$ at $200 \mathrm{~km}$ of altitude to $\sim 8 \times 10^{7}$ photons $\cdot \mathrm{cm}^{-2} \cdot \mathrm{s}^{-1}$ at $70 \mathrm{~km}$ of altitude (Gudipati et al., 2013; Wilson and Atreya, 2004). According to the model of Larson et al. 2014, aerosols spend most of their time in the lower atmosphere. Therefore, we can use the total lifetime, and the flux at $200 \mathrm{~km}$, which is the higher limit of the photon flux received at this wavelength in the stratosphere and the troposphere of Titan to calculate an upper limit of the fluence received by ices coated on aerosols. We obtained a value of $2.7 \times 10^{21}$ photons $\cdot \mathrm{cm}^{-2}$ at $350 \mathrm{~nm}$ to be delivered to an aerosol before reaching Titan 
surface, the same order of magnitude as the one used in our experiment at $355 \mathrm{~nm}$ (i.e. $1.8 \times 10^{21}$

photons $\cdot \mathrm{cm}^{-2}$ ). It is likely that the photon fluence received by an ice particle in Titan atmosphere would be lower than this upper limit. Indeed, we should keep in mind that the photon flux at 350 $\mathrm{nm}$ decreases by several orders of magnitude with the altitude in the lower atmospheric layers as these particles travel from the stratosphere to the troposphere and on to the surface. Further, our data is obtained for a given monochromatic laser wavelength and our data needs to be integrated to represent the range of wavelengths reaching the lower atmosphere and the surface of Titan, which could increase the photodesorption yields - but not photochemistry yields - based on the data presented in Figure 8. Consequently, photodesorption could open up a replenishing channel for a part of the acetylene ice to be converted into the gas-phase and fed into Titan's atmosphere, even if further quantification is necessary to better understand the importance of this process. Thus, it is important to include photodesorption to describe the microphysics of Titan's aerosols, particularly involving highly-abundant and highly-volatile ices such as acetylene. Finally, similar processes could be occurring with other condensed volatiles in Titan's ice particles, aerosols, and on the surface.

\subsection{Photochemistry of acetylene on tholins}

We have demonstrated that tholins could drive photodesorption of coated solid acetylene, while irradiated at UV-Vis wavelengths where the tholins have a strong absorption, but not acetylene itself. In the case of the experiments conducted with the $T_{1}$-tholin samples and irradiated at $355 \mathrm{~nm}$, the amount of acetylene desorbed, as quantified by mass spectrometry, cannot account for the total amount of acetylene consumed as determined by infrared spectroscopy. Another process has to be responsible for the rest of the acetylene ( $75 \%)$ consumed in these experiments. Direct solid-state photochemistry of $\mathrm{C}_{2} \mathrm{H}_{2}$ required higher energy photons than the $355 \mathrm{~nm}$ used in this experiment, as absorption of the acetylene (for the first excited $\mathrm{S}_{0}-\mathrm{S}_{1}$ state) starting at wavelength shorter than $237 \mathrm{~nm}$ (Couturier-Tamburelli et al., 2015; Malsch et al., 2001). Moreover, the direct acetylene ice photochemistry in the VUV has been experimentally studied, highlighting the formation of longer hydrocarbons molecules, including the formation of polymer (Compagnini et al., 2009; Cuylle et al., 2014). They were not observed in our experiments, excluding direct photochemistry in the acetylene layer, also in agreement with our observation that no significant depletion of $\mathrm{C}_{2} \mathrm{H}_{2}$ was detected when pure $\mathrm{C}_{2} \mathrm{H}_{2}$ was deposited on a sapphire window. This leaves the possibility for photochemical 
reactions of acetylene with the tholins further propagating into the crystalline $\mathrm{C}_{2} \mathrm{H}_{2}$ ice layer as shown in a previous experimental study for nitrile compounds (Couturier-Tamburelli et al., 2018). In order to explore this possibility, we analyzed infrared spectra obtained before the beginning of the experiment and subsequent to warming up of the photolyzed $\mathrm{C}_{2} \mathrm{H}_{2}$ - $\mathrm{T}_{1}$-tholin film at $300 \mathrm{~K}$, as shown in Figure 10. We should note here that the tholin film thickness is approximately $600 \mathrm{~nm}$, whereas the acetylene film thickness is $\sim 15-20 \mathrm{~nm}$, of which $60 \%$ photodepleted ( $15 \%$ photodesorption and $45 \%$ photochemistry), resulting in $\sim 1 \%$ material addition to the tholin at the end of this experiment.

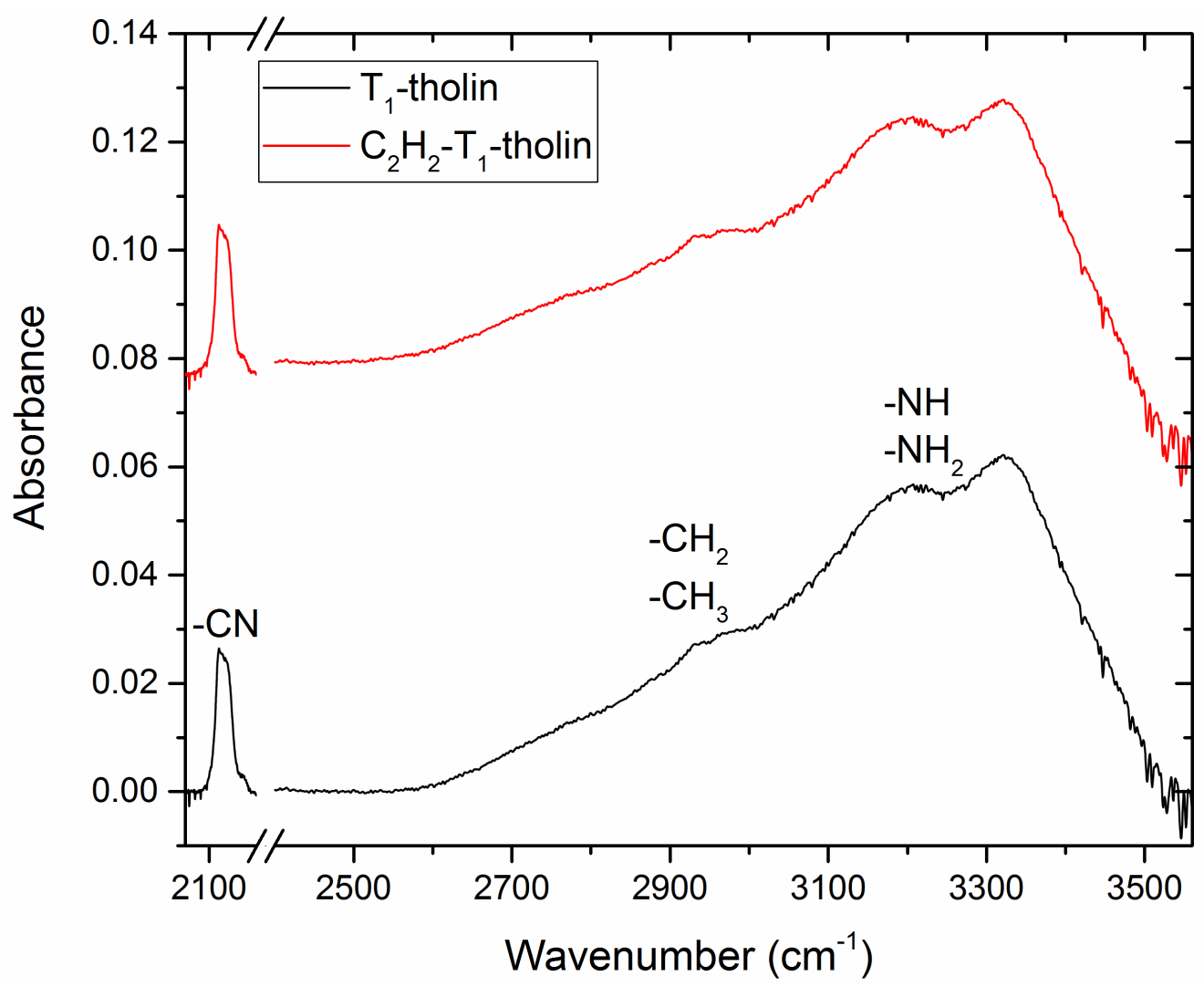

Figure 10: Infrared spectra of the T1-tholin sample at room temperature before the irradiation (black) and subsequent to warming up the photolyzed $\mathrm{C}_{2} \mathrm{H}_{2}-\mathrm{T}_{1}$-tholin (red).

From the spectra shown in Figure 10, we did not find significant changes in these spectra. It is likely because while acetylene $\mathrm{C}-\mathrm{H}$ infrared absorption band-strength is very high, when incorporated into the tholin film covalently, the resulting $\mathrm{C}-\mathrm{H}$ absorption may not be of the same band-strength and that the environment in which such bonding occurred may vary, resulting in very broad new absorbance of the photochemically incorporated $\mathrm{C}_{2} \mathrm{H}_{2}$ in the $\mathrm{T}_{1}$-tholin. Indeed, 
polymer IR absorptions tend to much broader and the new signals may be buried under the broad polymer absorption. Thus, infrared spectra did not yield convincingly concrete new information on the potential chemical structures of incorporated $\mathrm{C}_{2} \mathrm{H}_{2}$. Our experiments using isotopically labeled acetylene $\left({ }^{13} \mathrm{C}_{2} \mathrm{H}_{2}\right)$ also did not yield any outstanding new features in the roomtemperature infrared spectrum that could have been assigned to incorporation of ${ }^{13} \mathrm{C}-\mathrm{H}$ into the tholins, again most likely due to broad absorption features and small isotope shifts between ${ }^{12} \mathrm{C}$ $\mathrm{H}$ and ${ }^{13} \mathrm{C}-\mathrm{H}$ frequencies being merely $10 \mathrm{~cm}^{-1}$. However, we observed a slight change in the broad structure around $3100 \mathrm{~cm}^{-1}$ between before and after photoreactivity of $\mathrm{C}_{2} \mathrm{H}_{2}$ with $\mathrm{T}_{1}$ tholin such that after the photolysis the vertical gap between the $-\mathrm{CH}_{2} /-\mathrm{CH}_{3}$ absorption around $2900 \mathrm{~cm}^{-1}$ and the peak for $-\mathrm{NH} /-\mathrm{NH}_{2}$ at $3200 \mathrm{~cm}^{-1}$ decreased. This could be due to the expected broad absorption due to the incorporated acetylene that could have resulted in $\mathrm{H}_{2} \mathrm{C}=\mathrm{HC}-\mathrm{N}$ (tholin) after reacting with an amine functional group. New experiments that are highly sensitive to surface chemical composition at a scale of a few nanometers are necessary to better understand the reactivity occurring at the interface between the tholins and the ice layer and the possible photochemical modifications in tholins - the laboratory analogs of Titan's atmospheric aerosols.

\section{Conclusion}

We have carried out a study of the photo-induced processes of acetylene ice film coated on laboratory analogues of Titan's aerosols - tholins - at wavelengths longer than $300 \mathrm{~nm}$. We have demonstrated that the aerosols can act as antennae by absorbing the photons in the UV-Vis wavelength regions and efficiently drive the photodepletion of accreted acetylene, $60 \%$ of the initial acetylene was depleted after a fluence of $1.8 \times 10^{21}$ photons $\cdot \mathrm{cm}^{-2}$ at $355 \mathrm{~nm}$.

Monitoring of the residual gases in the vacuum chamber by mass spectrometry has allowed us to determine that $15 \%$ of the initial acetylene was photodesorbed. This is an indirect process driven by the absorption of photons first by tholins at the wavelength used for the irradiation and subsequently transferring that energy to accreted acetylene ice. We have calculated a photodesorption rate of acetylene to be $(2.1 \pm 0.2) \times 10^{-6}$ molecules photon ${ }^{-1}$ averaged on the total irradiation time at $355 \mathrm{~nm}$. The photon fluences used in our experiment are an upper limit for the one received at $355 \mathrm{~nm}$ by the aerosols during their sedimentation through the stratosphere and the troposphere of Titan, where condensed species are observed. Integrated photon flux at 
different altitudes of Titan's atmosphere would help better quantification of the photodesorption efficiencies. To better constrain the importance of this process, it would be necessary to extend our studies to a range of photon wavelengths $(>250 \mathrm{~nm})$, from the altitudes where $\mathrm{C}_{2} \mathrm{H}_{2}$ condenses $(\sim 70 \mathrm{~km})$ down to the surface, and then model their fluences at various altitudes in Titan's lower atmosphere.

We found that the photodesorption process alone could not explain all of the acetylene photodepletion that was measured. Since the direct solid-state photochemistry of solid acetylene is not possible at $355 \mathrm{~nm}$, acetylene should react photochemically with the $\mathrm{T}_{1}$-tholins during the energy transfer process, where unsaturated bonds and amine functional groups become easy locations for acetylene reaction with tholins. New and more sensitive surface analysis methods that can interrogate the upper few nanometers are needed to confirm our hypothesis that tholins first absorb photons and transfer this energy to $\mathrm{C}_{2} \mathrm{H}_{2}$ molecules for desorption or initiate chemical bonding through excited-state photochemistry between unsaturated tholin centers and acetylene.

\section{Acknowledgments}

This work is supported by the NASA Solar System Workings grant "Photochemistry in Titan's Lower Atmosphere". The research work has been carried out at the Jet Propulsion Laboratory, California Institute of Technology under a contract with the National Aeronautics and Space Administration. NC thanks the European Research Council for funding via the ERC PrimChem project (grant agreement No. 636829.). We thank Dr. Bryana Henderson for proofreading the manuscript.

\section{References}

Anderson, C. M., Samuelson, R. E., 2011. Titan's aerosol and stratospheric ice opacities between 18 and $500 \mu \mathrm{m}$ : Vertical and spectral characteristics from Cassini CIRS. Icarus. 212, 762778.

Anderson, C. M., Samuelson, R. E., Bjoraker, G. L., Achterberg, R. K., 2010. Particle size and abundance of $\mathrm{HC}_{3} \mathrm{~N}$ ice in Titan's lower stratosphere at high northern latitudes. Icarus. 207, 914-922.

Anderson, C. M., Samuelson, R. E., Yung, Y. L., McLain, J. L., 2016. Solid-state photochemistry as a formation mechanism for Titan's stratospheric $\mathrm{C}_{4} \mathrm{~N}_{2}$ ice clouds. Geophysical Research Letters. 43, 3088-3094.

Barth, E. L., 2017. Modeling survey of ices in Titan's stratosphere. Planetary and Space Science. 137, 20-31. 
Barth, E. L., Toon, O. B., 2003. Microphysical modeling of ethane ice clouds in titan's atmosphere. Icarus. 162, 94-113.

Bertin, M., et al., 2013. Indirect Ultraviolet Photodesorption from CO: $\mathrm{N}_{2}$ Binary Ices - an Efficient Grain-gas Process. The Astrophysical Journal. 779, 120.

Bertin, M., et al., 2012. UV photodesorption of interstellar CO ice analogues: from subsurface excitation to surface desorption. Physical Chemistry Chemical Physics. 14, 9929-9935.

Brassé, C., Muñoz, O., Coll, P., Raulin, F., 2015. Optical constants of Titan aerosols and their tholins analogs: Experimental results and modeling/observational data. Planetary and Space Science. 109-110, 159-174.

Cable, M. L., Hörst, S. M., Hodyss, R., Beauchamp, P. M., Smith, M. A., Willis, P. A., 2011. Titan Tholins: Simulating Titan Organic Chemistry in the Cassini-Huygens Era. Chemical Reviews. 112, 1882-1909.

Carrasco, N., et al., 2009. Chemical Characterization of Titan's Tholins: Solubility, Morphology and Molecular Structure Revisited $\dagger$. The Journal of Physical Chemistry A. 113, 1119511203.

Clark, R. N., et al., 2010. Detection and mapping of hydrocarbon deposits on Titan. Journal of Geophysical Research: Planets. 115.

Compagnini, G., D’Urso, L., Puglisi, O., Baratta, G. A., Strazzulla, G., 2009. The irradiation of solid hydrocarbons and the formation of linear carbon chains. Carbon. 47, 1605-1607.

Coustenis, A., Schmitt, B., Khanna, R. K., Trotta, F., 1999. Plausible condensates in Titan's stratosphere from Voyager infrared spectra. Planetary and Space Science. 47, 1305-1329.

Couturier-Tamburelli, I., Gudipati, M. S., Lignell, A., Jacovi, R., Piétri, N., 2014. Spectroscopic studies of non-volatile residue formed by photochemistry of solid $\mathrm{C}_{4} \mathrm{~N}_{2}$ : A model of condensed aerosol formation on Titan. Icarus. 234, 81-90.

Couturier-Tamburelli, I., Piétri, N., Gudipati, M. S., 2015. Simulation of Titan's atmospheric photochemistry. A\&A. 578, A111.

Couturier-Tamburelli, I., Piétri, N., Le Letty, V., Chiavassa, T., Gudipati, M., 2018. UV-Vis Light-induced Aging of Titan's Haze and Ice. The Astrophysical Journal. 852, 117.

Cui, J., et al., 2009. Analysis of Titan's neutral upper atmosphere from Cassini Ion Neutral Mass Spectrometer measurements. Icarus. 200, 581-615.

Cuylle, S. H., Zhao, D., Strazzulla, G., Linnartz, H., 2014. Vacuum ultraviolet photochemistry of solid acetylene: a multispectral approach. A\&A. 570, A83.

de Kok, R., Irwin, P. G. J., Teanby, N. A., 2008. Condensation in Titan's stratosphere during polar winter. Icarus. 197, 572-578.

de Kok, R. J., Teanby, N. A., Maltagliati, L., Irwin, P. G. J., Vinatier, S., 2014. HCN ice in Titan's high-altitude southern polar cloud. Nature. 514, 65-67.

Fayolle, E. C., et al., 2011. CO Ice Photodesorption: A Wavelength-dependent Study. The Astrophysical Journal Letters. 739, L36.

Fillion, J. H., et al., 2014. Wavelength resolved UV photodesorption and photochemistry of $\mathrm{CO}_{2}$ ice. Faraday Discussions. 168, 533-552.

Flasar, F. M., et al., 2005. Titan's Atmospheric Temperatures, Winds, and Composition. Science. 308, 975-978.

Frère, C., Raulin, F., Israel, G., Cabane, M., 1990. Microphysical modeling of titan's aerosols: Application to the in situ analysis. Advances in Space Research. 10, 159-163.

Fulchignoni, M., et al., 2005. In situ measurements of the physical characteristics of Titan's environment. Nature. 438, 785-791. 
Gautier, T., et al., 2012. Mid- and far-infrared absorption spectroscopy of Titan's aerosols analogues. Icarus. 221, 320-327.

Gautier, T., et al., 2014. Nitrogen incorporation in Titan's tholins inferred by high resolution orbitrap mass spectrometry and gas chromatography-mass spectrometry. Earth and Planetary Science Letters. 404, 33-42.

Griffith, C. A., Doose, L., Tomasko, M. G., Penteado, P. F., See, C., 2012. Radiative transfer analyses of Titan's tropical atmosphere. Icarus. 218, 975-988.

Griffith, C. A., Owen, T., Geballe, T. R., Rayner, J., Rannou, P., 2003. Evidence for the Exposure of Water Ice on Titan's Surface. Science. 300, 628-630.

Gudipati, M. S., Jacovi, R., Couturier-Tamburelli, I., Lignell, A., Allen, M., 2013. Photochemical activity of Titan's low-altitude condensed haze. Nat Commun. 4, 1648.

Hayne, P. O., McCord, T. B., Sotin, C., 2014. Titan's surface composition and atmospheric transmission with solar occultation measurements by Cassini VIMS. Icarus. 243, 158172.

Hörst, S. M., 2017. Titan's atmosphere and climate. Journal of Geophysical Research: Planets. $122,432-482$.

Hudson, R. L., Ferrante, R. F., Moore, M. H., 2014. Infrared spectra and optical constants of astronomical ices: I. Amorphous and crystalline acetylene. Icarus. 228, 276-287.

Khanna, R. K., 2005a. Condensed species in Titan's atmosphere: Identification of crystalline propionitrile $\left(\mathrm{C}_{2} \mathrm{H}_{5} \mathrm{CN}, \mathrm{CH}_{3} \mathrm{CH}_{2} \mathrm{CN}\right)$ based on laboratory infrared data. Icarus. 177, 116121.

Khanna, R. K., 2005b. Condensed species in Titan's stratosphere: Confirmation of crystalline cyanoacetylene $\left(\mathrm{HC}_{3} \mathrm{~N}\right)$ and evidence for crystalline acetylene $\left(\mathrm{C}_{2} \mathrm{H}_{2}\right)$ on Titan. Icarus. 178, 165-170.

Khanna, R. K., Perera-Jarmer, M. A., Ospina, M. J., 1987. Vibrational infrared and raman spectra of dicyanoacetylene. Spectrochimica Acta Part A: Molecular Spectroscopy. 43, 421-425.

Knez, C., Moore, M. H., Ferrante, R. F., Hudson, R. L., 2012. Laboratory IR Studies and Astrophysical Implications of $\mathrm{C}_{2} \mathrm{H}_{2}$-Containing Binary Ices. The Astrophysical Journal. 748, 95.

Koskinen, T. T., et al., 2011. The mesosphere and lower thermosphere of Titan revealed by Cassini/UVIS stellar occultations. Icarus. 216, 507-534.

Larson, E. J. L., Toon, O. B., Friedson, A. J., 2014. Simulating Titan's aerosols in a three dimensional general circulation model. Icarus. 243, 400-419.

Lavvas, P., Griffith, C. A., Yelle, R. V., 2011a. Condensation in Titan's atmosphere at the Huygens landing site. Icarus. 215, 732-750.

Lavvas, P., Sander, M., Kraft, M., Imanaka, H., 2011b. Surface Chemistry and Particle Shape: Processes for the Evolution of Aerosols in Titan's Atmosphere. The Astrophysical Journal. 728, 80.

Lavvas, P., Yelle, R. V., Griffith, C. A., 2010. Titan's vertical aerosol structure at the Huygens landing site: Constraints on particle size, density, charge, and refractive index. Icarus. 210, 832-842.

Lavvas, P., et al., 2013. Aerosol growth in Titan's ionosphere. Proceedings of the National Academy of Sciences. 110, 2729-2734. 
Lavvas, P. P., Coustenis, A., Vardavas, I. M., 2008. Coupling photochemistry with haze formation in Titan's atmosphere, Part II: Results and validation with Cassini/Huygens data. Planetary and Space Science. 56, 67-99.

Liang, M.-C., Yung, Y. L., Shemansky, D. E., 2007. Photolytically Generated Aerosols in the Mesosphere and Thermosphere of Titan. The Astrophysical Journal Letters. 661, L199.

Mahjoub, A., Carrasco, N., Dahoo, P. R., Gautier, T., Szopa, C., Cernogora, G., 2012. Influence of methane concentration on the optical indices of Titan's aerosols analogues. Icarus. 221, 670-677.

Malsch, K., Hohlneicher, G., Schork, R., Koppel, H., 2001. A quantum dynamical examination of the vibronic structure of singlet and triplet spectra of acetylene. Physical Chemistry Chemical Physics. 3, 5393-5407.

Mayo, L. A., Samuelson, R. E., 2005. Condensate clouds in Titan's north polar stratosphere. Icarus. 176, 316-330.

McCord, T. B., et al., 2006. Composition of Titan's surface from Cassini VIMS. Planetary and Space Science. 54, 1524-1539.

McCord, T. B., et al., 2008. Titan's surface: Search for spectral diversity and composition using the Cassini VIMS investigation. Icarus. 194, 212-242.

McMullan, R. K., Kvick, A., Popelier, P., 1992. Structures of cubic and orthorhombic phases of acetylene by single-crystal neutron diffraction. Acta Crystallographica Section B. 48, 726-731.

Niemann, H. B., et al., 2010. Composition of Titan's lower atmosphere and simple surface volatiles as measured by the Cassini-Huygens probe gas chromatograph mass spectrometer experiment. Journal of Geophysical Research: Planets. 115, E12006.

Rannou, P., et al., 2016. Titan's surface spectra at the Huygens landing site and Shangri-La. Icarus. 270, 291-306.

Raulin, F., Owen, T., 2002. Organic Chemistry and Exobiology on Titan. Space Science Reviews. 104, 377-394.

Sagan, C., Reid Thompson, W., 1984. Production and condensation of organic gases in the atmosphere of Titan. Icarus. 59, 133-161.

Samuelson, R. E., A. Mayo, L., 1991. Thermal infrared properties of Titan's stratospheric aerosol. Icarus. 91, 207-219.

Samuelson, R. E., Mayo, L. A., Knuckles, M. A., Khanna, R. J., 1997. C ${ }_{4} \mathrm{~N}_{2}$ ice in Titan's north polar stratosphere. Planetary and Space Science. 45, 941-948.

Samuelson, R. E., Smith, M. D., Achterberg, R. K., Pearl, J. C., 2007. Cassini CIRS update on stratospheric ices at Titan's winter pole. Icarus. 189, 63-71.

Sciamma-O'Brien, E., Carrasco, N., Szopa, C., Buch, A., Cernogora, G., 2010. Titan's atmosphere: An optimal gas mixture for aerosol production? Icarus. 209, 704-714.

Singh, S., et al., 2016. Acetylene on Titan's Surface. The Astrophysical Journal. 828, 55.

Szopa, C., Cernogora, G., Boufendi, L., Correia, J. J., Coll, P., 2006. PAMPRE: A dusty plasma experiment for Titan's tholins production and study. Planetary and Space Science. 54, 394-404.

Thrower, J. D., et al., 2008. Desorption of Hot Molecules from Photon Irradiated Interstellar Ices. The Astrophysical Journal. 673, 1233.

Tomasko, M. G., et al., 2005. Rain, winds and haze during the Huygens probe's descent to Titan's surface. Nature. 438, 765-778. 
Vinatier, S., et al., 2010. Analysis of Cassini/CIRS limb spectra of Titan acquired during the nominal mission: I. Hydrocarbons, nitriles and $\mathrm{CO}_{2}$ vertical mixing ratio profiles. Icarus. 205, 559-570.

Vuitton, V., Yelle, R. V., Cui, J., 2008. Formation and distribution of benzene on Titan. Journal of Geophysical Research: Planets. 113, E05007.

Waite, J. H., et al., 2007. The Process of Tholin Formation in Titan's Upper Atmosphere. Science. 316, 870-875.

Wilson, E. H., Atreya, S. K., 2004. Current state of modeling the photochemistry of Titan's mutually dependent atmosphere and ionosphere. Journal of Geophysical Research: Planets. 109, E06002. 